\title{
A Two-Tiered In Vitro Approach to De-Risk Drug Candidates for Potential Bile Salt Export Pump Inhibition Liabilities in Drug Discovery ${ }^{\mathbb{S}}$
}

\author{
Michael J. Hafey, Robert Houle, Keith Q. Tanis, lan Knemeyer, Jackie Shang, ${ }^{1}$ Qing Chen, ${ }^{2}$ \\ Andreas Baudy, James Monroe, Frank D. Sistare, and Raymond Evers ${ }^{3}$
}

Departments of Pharmacokinetics, Pharmacodynamics \& Drug Metabolism (PPDM) (M.J.H., R.H., I.K., J.S., Q.C., R.E.), Genetics and Pharmacogenomics (K.Q.T.), and Safety Assessment and Laboratory Animal Resources (SALAR) (A.B., J.M., F.D.S.), Merck \& Co., Inc., Kenilworth, New Jersey

Received April 17, 2020; accepted August 3, 2020

\begin{abstract}
Hepatocellular accumulation of bile salts by inhibition of bile salt export pump (BSEP/ABCB11) may result in cholestasis and is one proposed mechanism of drug-induced liver injury (DILI). To understand the relationship between BSEP inhibition and DILI, we evaluated 64 DILI-positive and 57 DILI-negative compounds in BSEP, multidrug resistance protein (MRP) 2, MRP3, and MRP4 vesicular inhibition assays. An empirical cutoff $(5 \mu \mathrm{M})$ for BSEP inhibition was established based on a relationship between BSEP $\mathrm{IC}_{50}$ values and the calculated maximal unbound concentration at the inlet of the human liver $\left(\mathrm{fu}^{\star} \mathrm{I}_{\mathrm{in}, \max }\right.$, assay specificity $=98 \%$ ). Including inhibition of MRP2-4 did not increase DILI predictivity. To further understand the potential to inhibit bile salt transport, a selected subset of $\mathbf{3 0}$ compounds were tested for inhibition of taurocholate (TCA) transport in a long-term human hepatocyte micropatterned co-culture (MPCC) system. The resulting IC $_{50}$ for TCA in vitro biliary clearance and biliary excretion index (BEI) in MPCCs were compared with the compound's $\mathrm{fu}^{*} \mathrm{I}_{\mathrm{in} \text {,max }}$ to assess potential risk for bile salt transport perturbation. The data show high
\end{abstract}

specificity (89\%). Nine out of 15 compounds showed an IC $\mathrm{I}_{50}$ value in the BSEP vesicular assay of $<5 \mu \mathrm{M}$, but the BEI IC ${ }_{50}$ was more than 10 -fold the $\mathrm{fu}^{*} \mathrm{l}_{\mathrm{in} \text {,max }}$, suggesting that inhibition of BSEP in vivo is unlikely. The data indicate that although BSEP inhibition measured in membrane vesicles correlates with DILI risk, that measurement of this assay activity is insufficient. A two-tiered strategy incorporating MPCCs is presented to reduce BSEP inhibition potential and improve DILI risk.

\section{SIGNIFICANCE STATEMENT}

This work describes a two-tiered in vitro approach to de-risk compounds for potential bile salt export pump inhibition liabilities in drug discovery utilizing membrane vesicles and a long-term human hepatocyte micropatterned co-culture system. Cutoffs to maximize specificity were established based on in vitro data from a set of 121 DILI-positive and -negative compounds and associated calculated maximal unbound concentration at the inlet of the human liver based on the highest clinical dose.

\section{Introduction}

Drug-induced liver injury (DILI) is the single most common adverse event leading to withdrawal of pharmaceuticals from the market and is a major reason for failure of drug candidates in development (Holt and $\mathrm{Ju}, 2010)$. Currently, DILI is difficult to predict with existing preclinical or in vitro models, with only half of drugs that cause human

This research was supported by Merck \& Co., Inc., Kenilworth, NJ, and no external funding was received.

${ }^{1}$ Current affiliation: Janssen Pharmaceuticals, Johnson \& Johnson, Springhouse, Pennsylvania.

${ }^{2}$ Current affiliation: Vertex Pharmaceuticals, Boston, Massachusetts.

${ }^{3}$ Current affiliation: Janssen Pharmaceuticals, Johnson \& Johnson, Springhouse, Pennsylvania.

https://doi.org/10.1124/dmd.120.000086.

S This article has supplemental material available at dmd.aspetjournals.org. hepatotoxicity being identified during preclinical testing (Sistare et al., 2016). Furthermore, the causes of DILI are likely multifactorial: as such, there is no single assay available to de-risk for DILI at the preclinical stage. Instead, a battery of assays is used with a "weight of evidence" assessment (Shah et al., 2015). In vitro assays have been established to examine potential mechanisms, including measurements of drug bioactivation, mitochondrial toxicity, and disruption of bile salt transport (Sistare et al., 2016; (Xu et al., 2019); Kang et al., 2020; Monroe et al., 2020).

Over the past decade, extensive research has been conducted to gain a better mechanistic understanding of disruption of bile salt transport and its relationship with DILI risk. The enterohepatic circulation of bile salts is complex, with intestinal and hepatic membrane transporters playing a critical role in bile salt homeostasis (Yang et al., 2013). In humans, uptake of bile salts from the plasma into the hepatocyte is mediated by the sodium taurocholate cotransporting polypeptide (NTCP) and organic

ABBREVIATIONS: AG, acyl-glucuronide; BEI, biliary excretion index; BSEP, bile salt export pump; CDF, 5-carboxy-2',7' dichlorofluorescein; $\mathrm{Cl}_{\text {biliary }}$, in vitro biliary clearance; $\mathrm{C}_{\max , \mathrm{ss}}$, steady-state $\mathrm{C}_{\max } ; \mathrm{CsA}$, cyclosporin $\mathrm{A}$; DILI, drug-induced liver injury; EA-SG, ethacrynic acid glutathione conjugate; $\mathrm{E}_{2} 17 \beta \mathrm{G}$, estradiol-17 $\beta$-D-glucuronide; fu $\mathrm{f}_{\mathrm{in} \text {,max }}$, maximal unbound concentration at the inlet of the human liver; HBSS, Hanks' balanced salt solution; MPCC, micropatterned co-culture; MRP, multidrug resistance protein; MS, mass spectrometry; NTCP, sodium taurocholate cotransporting polypeptide; OATP, organic anion transporting polypeptide; TCA, taurocholate. 


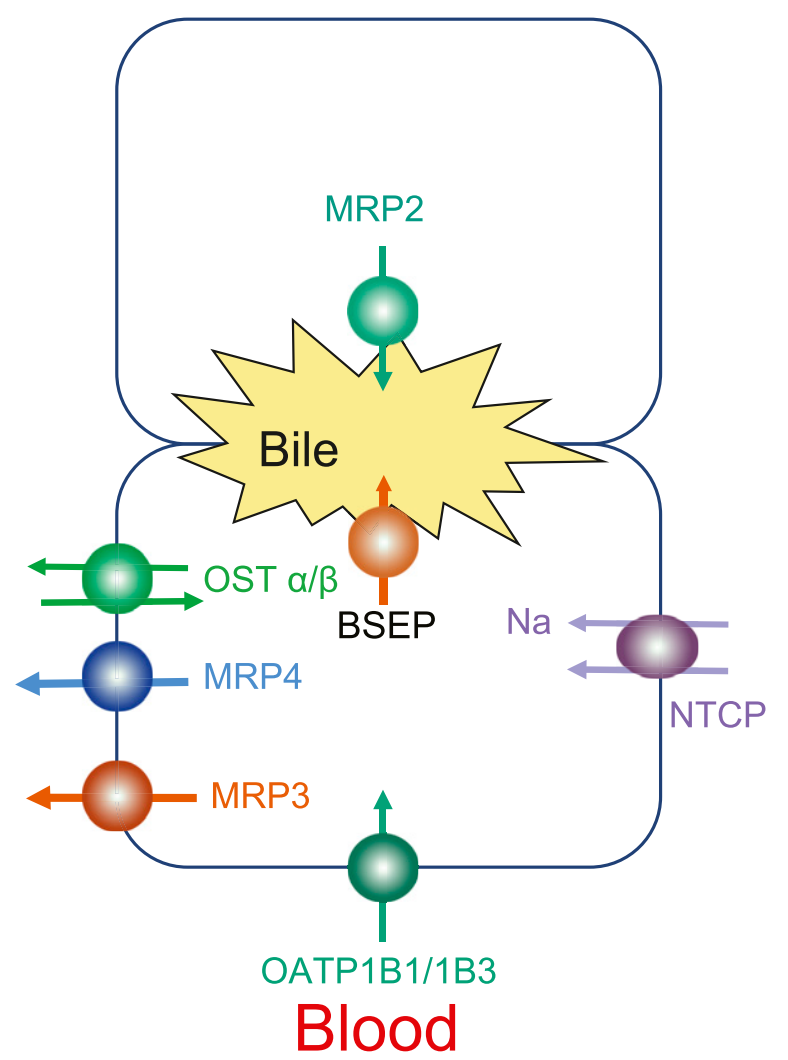

Fig. 1. The role of transporters in the enterohepatic circulation of bile salts.

anion transporting polypeptides (OATPs) 1B1 and 1B3, whereas efflux of bile salts from the hepatocyte into the bile is mediated mainly by bile salt export pump (BSEP), the rate-limiting step in bile salt transport, and to some extent by the multidrug resistance protein (MRP) 2 (Yang et al., 2013), Fig. 1).

BSEP-mediated efflux represents the driving force for the generation of bile salt-dependent bile flow, and thus, inhibition of BSEP may lead to elevations in intracellular bile salts in hepatocytes which can contribute to DILI (Kenna et al., 2018). Compensatory transport mechanisms, including downregulation of the sinusoidal uptake transporters (NTCP/OATP) and upregulation of both sinusoidal (MRP3, MRP4, organic solute transporter (OST) $\alpha / \beta$ ) and canalicular efflux transporters (BSEP/MRP2), primarily through farnesoid $\mathrm{X}$ receptor (FXR) signaling, aid in maintaining cellular bile salt homeostasis (Rodrigues et al., 2014).

To increase understanding of the relationship between BSEP inhibition and DILI, several groups have examined inhibition of BSEPmediated transport of taurocholic acid (TCA) by drugs in a vesicular BSEP inhibition assay ((Morgan et al., 2010); Dawson et al., 2012; Pedersen et al., 2013; Yucha et al., 2017). These studies show that several drugs implicated in DILI inhibit BSEP, but that there were false positives (i.e., BSEP inhibitors that were not DILI positive varied from $7 \%$ to $38 \%$ ). $\mathrm{IC}_{50}$ cutoffs used to define BSEP inhibitors of concern varied widely, ranging from $<25 \mu \mathrm{M}$ (Morgan et al., 2010) to $<300 \mu \mathrm{M}$ (Dawson et al., 2012). In addition, differences in compound test sets, adjudication of compounds as DILI positive, and in vitro inhibition values added additional complexity. False positives can also be due to the fact that membrane vesicles lack the metabolic and transport mechanisms needed to assess metabolites and/or interplay with other bile acid transporters in hepatocytes. Thus, further evaluation of compounds found to inhibit BSEP in vesicles is warranted in a hepatocyte-based model. Several groups have used sandwich-cultured hepatocytes to assess drug effects on hepatobiliary bile acid disposition (Yang et al., 2016), but to our knowledge no comprehensive comparison has been conducted between DILI-positive and DILI-negative compounds in both vesicles and a hepatocyte-based model.

Human hepatocyte micropatterned co-cultures (MPCCs) are a bioengineered microliver platform in which human hepatocytes and mouse fibroblasts are plated in co-culture and in which stable expression of phase I and II enzymes and several transporters is maintained over several weeks in culture (Khetani and Bhatia, 2008). Furthermore, functional OATP, NTCP, and MRP2 activity has been shown (Khetani and Bhatia, 2008; (Ramsden et al., 2014)). Here, we explored whether MPCCs may serve as a platform to evaluate the effect of test compounds on bile salt transport and whether compounds that inhibit BSEP in membrane vesicles also inhibit canalicular efflux in MPCCs. Furthermore, we sought to understand the relationship between in vitro BSEP, MRP2, MRP3, and MRP4 inhibition, liver exposure, and human DILI risk prediction by examining the inhibition potential of 64 DILI-positive and 57 DILI-negative compounds on these transporters. For a subset of 30 compounds (19 DILI positive and 11 DILI negative), the inhibition of the uptake and efflux of TCA in MPCCs was evaluated to test the hypothesis that BSEP inhibition in vesicles results in a decreased biliary excretion index (BEI) in MPCCs.

\section{Methods}

Materials. Membrane vesicles containing human BSEP, MRP2, MRP3, or MRP4 were manufactured by Genomembrane (Kanagawa, Japan) and purchased through Life Technologies (Grand Island, NY). $\left[{ }^{3} \mathrm{H}\right]$ TCA and $\left[{ }^{3} \mathrm{H}\right]$-estradiol-17 $\beta$-D-glucuronide $\left(\mathrm{E}_{2} 17 \beta \mathrm{G}\right)$ were purchased from Perkin Elmer. $\left[{ }^{3} \mathrm{H}\right]$-Folic acid was purchased from Moravek, Inc. (Brea, CA). $\left[{ }^{14} \mathrm{C}\right]$-Ethacrynic acid glutathione conjugate (EA-SG) and all MK compounds included in this work were synthesized by Merck \& Co., Inc. (Kenilworth, NJ). Test compounds were purchased from a variety of commercial sources (as referenced in (Monroe et al., 2020)). All other reagents were commercially obtained and were of the highest grade possible.

Classification of DILI-Positive and -Negative Compounds. The list of drugs included in this study and their DILI-positive or -negative categorical assignments are provided in Table 1 as referenced in Monroe et al. (Monroe et al., 2020). Several sources were referenced to make judgments on liver safety for drugs used for this assessment, including clinical case reports from published literature, product labels, the National Institutes of Health LiverTox data base, and published country registries. Marketed drugs labeled with more moderate and non-life-threatening increases in aminotransferases, representing a reversible and adaptive response to drug treatment, and not documented to be accompanied by acute liver failure as an indication of permanent injury to the liver, were not classified as DILI-positive clinical hepatotoxicants. Market exposure over time as well as daily dose were also considered, as drugs that are administered safely at relatively high doses resulting in significant daily liver dose burdens without liver injury support the classification of nontoxicants. True clinical hepatotoxicants were identified for testing among marketed or marketed-but-withdrawn compounds that have documented clinical diagnoses of acute liver failure. In addition, compounds were selected for inclusion in the test set that have been discontinued by pharmaceutical sponsors at clinical stages of drug development due to strong liver safety transaminase signals, but without allowing instances of acute liver failure. For such drugs, the liver safety signals were considered sufficiently strong for sponsors to discontinue development and so are representative of the type of test compounds that needs to be 
identified earlier, and therefore were also characterized as true clinical DILI positives in our analyses.

Inhibition of Transport by BSEP, MRP2, MRP3, and MRP4 in Membrane Vesicles. The inhibitory effect of test compounds on ATPdependent $\left[{ }^{3} \mathrm{H}\right]$ TCA $(1 \mu \mathrm{M}),\left[{ }^{14} \mathrm{C}\right]$ EA-SG $(1 \mu \mathrm{M}),\left[{ }^{3} \mathrm{H}\right] \mathrm{E}_{2} 17 \mathrm{BG}(1$ $\mu \mathrm{M})$, or $\left[{ }^{3} \mathrm{H}\right]$ folic acid $(10 \mu \mathrm{M})$ uptake was conducted in membrane vesicles isolated from Sf9 cells containing BSEP, MRP2, MRP3, and MRP4, respectively, using three test compound concentrations (1, 10, and $25 \mu \mathrm{M}$ ) as described previously (Prueksaritanont et al., 2014). Transporter-mediated uptake was calculated by subtracting the uptake of the probe substrate in the presence of AMP from that in the presence of ATP. Data were normalized to percentages of control, where uptake in the absence of test compound was $100 \%$. Estimated $\mathrm{IC}_{50}$ values for inhibition of transporter-mediated uptake were obtained by fitting the data using eq. 1 by nonlinear regression analysis using GraphPad Prism:

$$
\% \text { Control }=100 /\left(1+\left(\frac{\mathrm{I}^{\mathrm{Hill} \text { Slope }}}{\mathrm{IC} 50^{\text {Hill Slope }}}\right)\right),
$$

where "\%Control" represents transporter-mediated uptake measured in the presence of various concentrations of inhibitor to that in the absence of inhibitor and "I" represents nominal inhibitor concentration. Estimated $\mathrm{IC}_{50}$ values were extrapolated up to $100 \mu \mathrm{M}$. In instances when an estimated $\mathrm{IC}_{50}$ could not be estimated, the $\mathrm{IC}_{50}$ value was noted as $>100 \mu \mathrm{M}$. To assess the accuracy of the three-point $\mathrm{IC}_{50}$ values, estimated $\mathrm{IC}_{50}$ values for seven compounds, including bosentan, nefazodone, and troglitazone, were compared with literature values and found to be within 3-fold of the published values (Dawson et al., 2012; Morgan et al., 2013).

The resulting $\mathrm{IC}_{50}$ values were compared with the estimated unbound concentration of test compounds at the inlet of the human liver $\left(\mathrm{fu} * \mathrm{I}_{\mathrm{in}, \max }\right)$, which was calculated according to the approach described by Hirano et al. (Hirano et al. 2006) using the highest clinical dose prescribed or tested in the clinic, and corresponding published $\mathrm{C}_{\max }$ values obtained from a variety of sources, including PharmaPendium, American Hospital Formulary Service Drug Information (http://www.ahfsdruginformation.com), and drug labels (Table 1). Empirical cutoffs were then established to limit the number of false positives and maximize assay specificity. Specifically, a threshold was set for BSEP, MRP2, MRP3, and MRP4 according to eqs. 2-5, respectively:

$$
\begin{gathered}
(\log 10(1 / I C 50)+0.87) *(2+\log 10(F u * \operatorname{Iin}, \max ))=0.1 \\
(\log 10(1 / I C 50)+1.9) *(2+\log 10(F u * \operatorname{Iin}, \max ))=0.1 \\
(\log 10(1 / I C 50)+1.85) *(0.6+\log 10(F u * \operatorname{Iin}, \max ))=0.05 \\
(\log 10(1 / I C 50)+1.6) *(2.2+\log 10(F u * \operatorname{Iin}, \max ))=0.5
\end{gathered}
$$

where values above 0.1 (BSEP and MRP2), 0.05 (MRP3), and 0.5 (MRP4) indicate that inhibition of the transporter may be indicative of DILI risk.

Fluorescent Imaging of Biliary Canaliculi as Well as BSEP and MRP2 Localization in Human MPCCs. Human HepatoPac MPCCs plated in black-walled 96-well plates were purchased from Ascendance Bio (Medford, MA) and used for all imaging experiments. Live visualization of bile canaliculi was performed after incubation with $10 \mu \mathrm{M}$ carboxy-5-(and-6)-carboxy-2',7'-dichlorofluorescein diacetate for 20 minutes at $37^{\circ} \mathrm{C}$, followed by fluorescent imaging using an In Cell 2000 imager (GE Healthcare).

Immunostaining for BSEP and MRP2 was performed initially by fixation in $4 \%$ para-formaldehyde for 15 minutes. Wells were then washed two times with PBS before incubation with $1 \%$ Triton X-100 for 10 minutes, $1 \%$ bovine serum albumin in PBS for 30 minutes at $37^{\circ} \mathrm{C}$, and then with primary antibodies against BSEP (N-16 \#sc-17292; Santa Cruz; (Jara et al., 2009)) and MRP2 (\#MAB4148; Millipore; (Scheffer et al., 2000)) at 1:200 dilutions in PBS for 1 hour at $37^{\circ} \mathrm{C}$. Wells were then washed four times with PBS and incubated for 1 hour at $37^{\circ} \mathrm{C}$ with Alexa Fluor 488 goat anti-rabbit IgG (\#A11034; Life Tech) and Alexa Fluor 647 goat anti-mouse IgG (\#A21236; Life Tech) at 1:500 in 0.1\% Triton X-100. Wells were then washed four times with PBS prior to fluorescent imaging using a GE In Cell 2000 Imager.

Inhibition of Intrinsic In Vitro Biliary Clearance and Biliary Excretion Index in Human MPCCs. The inhibitory effect of the test compound on the intrinsic in vitro biliary clearance $\left(\mathrm{Cl}_{\text {biliary }}\right)$ and $\mathrm{BEI}$ of $\left[{ }^{3} \mathrm{H}\right]$ TCA $(1 \mu \mathrm{M})$ was conducted in human HepatoPac MPCCs at Ascendance Bio using a method similar to that used in sandwichcultured hepatocytes described in Wolf et al. (2010).

Cryopreserved human hepatocytes (Celsis, Baltimore, MD) and cryopreserved 3T3 J2 mouse fibroblasts (stromal cells) were used to create MPCCs as described in Ukairo et al. (2013). Hepatocytes were from a single male human donor (lot GC4008; Triangle Research Laboratories, Research Triangle Park, NC), which was selected based on the uptake and efflux windows obtained with TCA after screening five hepatocyte lots. Culture medium was replaced every 2 days for 8 days. All reagents used in the study were of analytical grade.

The transport of $\left[{ }^{3} \mathrm{H}\right] \mathrm{TCA}$ was measured in 96-well human MPCCs in the presence and absence of varying concentrations of test compound or cyclosporin A (CsA; positive control for transporter inhibition) after 10minute or 24-hour preincubation with test compound. Twenty-four-hour preincubations were carried out in hepatocyte culture medium without serum. Ten minutes prior to initiation of uptake/efflux assays, cells were washed three times in Hanks' balanced salt solution (HBSS) buffer containing calcium or HBSS devoid of calcium before incubation with buffers containing test compounds. MPCCs used to measure TCA uptake into the hepatocytes and efflux into the canalicular space were incubated in buffer containing calcium, whereas cells used to measure uptake into hepatocytes were incubated in buffer devoid of calcium for 10 minutes at $37^{\circ} \mathrm{C}$ (Wolf et al., 2010). All wells were then washed with HBSS containing calcium. Transport was initiated by the addition of TCA $(1 \mu \mathrm{M} ; 0.5 \mu \mathrm{Ci} / \mathrm{ml})$ in the presence or absence of various concentrations of test compound or CsA in HBSS containing calcium at $37^{\circ} \mathrm{C}$ for 10 minutes for uptake and efflux assessments. Transport was stopped by the addition of ice-cold PBS; cells were washed three times in ice-cold PBS and lysed (1\% Triton X-100 in PBS) for 20 minutes. Cell lysates were pipetted up and down several times and then transferred to scintillation vials or plates containing scintillation fluid. The radioactivity of each sample was measured using liquid scintillation counting. All compounds were analyzed in triplicate.

Protein was extracted from representative MPCC wells by lysing the cells in radioimmunoprecipitation assay (RIPA) buffer (Rockland Immunochemicals Inc., Gilbertsville, PA) for 5 minutes on ice. The amount of protein per well was measured using the Pierce BCA Protein Assay kit (Rockford, IL). The protein content of stromal-only cultures was also measured, and hepatic protein was calculated by subtracting stromal-only protein from MPCC protein values. The scintillation counts were then normalized by hepatic protein content. The experiment was performed in triplicate.

The $\mathrm{Cl}_{\text {biliary }}$ and BEI were calculated using the mean scintillation counts in eqs. 6 and 7 (Wolf et al., 2010):

$$
\begin{gathered}
\text { in vitro } \mathrm{Cl}_{\text {biliary }}=\frac{\text { accumulation }_{\text {cells }+ \text { bile }}-\text { accumulation }_{\text {cells }}}{A U C_{\text {medium }}} \\
B E I=\frac{\text { accumulation }_{\text {cells }+ \text { bile }}-\text { accumulation }_{\text {cells }}}{\text { accumulation }_{\text {cells }+ \text { bile }}} \times 100,
\end{gathered}
$$


TABLE 1

Estimated $\mathrm{IC}_{50}$ values for BSEP and MRP2-4 generated in membrane vesicles for DILI-negative and -positive compounds

Corresponding unbound liver inlet concentrations were calculated from the highest clinical dose and associated $\mathrm{C}_{\max }$.fu, fraction unbound

\begin{tabular}{|c|c|c|c|c|c|c|c|c|c|}
\hline \multirow{2}{*}{ Compound } & \multirow{2}{*}{$\begin{array}{c}\text { DILI } \\
\text { classification }\end{array}$} & \multicolumn{4}{|c|}{ Estimated $\mathrm{IC}_{50 \mu \mathrm{M}}$ ) } & \multirow{2}{*}{ Dose (mg) } & \multirow{2}{*}{$\mathrm{C}_{\max }(\mu \mathrm{M})$} & \multirow{2}{*}{ fu } & \multirow{2}{*}{$\mathrm{fu}^{*} \mathrm{I}_{\mathrm{in}, \max }(\mu \mathrm{M})$} \\
\hline & & BSEP & MRP2 & MRP3 & MRP4 & & & & \\
\hline Ambrisentan & Negative & $>100$ & $>100$ & $>100$ & $>100$ & 10 & 9.8 & 0.01 & 0.12 \\
\hline Amoxicillin & Negative & $>100$ & $>100$ & $>100$ & $>100$ & 500 & 4.93 & 0.83 & 79.80 \\
\hline Amprenavir & Negative & 98 & $>100$ & 45.7 & $>100$ & 1200 & 18 & 0.10 & 17.62 \\
\hline Aripiprazole & Negative & 42.1 & $>100$ & $>100$ & $>100$ & 15 & 0.13 & 0.01 & 0.02 \\
\hline Aspirin & Negative & $>100$ & $>100$ & 45 & $>100$ & 2000 & 77.7 & 0.004 & 3.27 \\
\hline Atorvastatin & Negative & 6.3 & $>100$ & 3.8 & 19.8 & 80 & 0.1 & 0.02 & 0.19 \\
\hline Buspirone & Negative & $>100$ & $>100$ & $>100$ & $>100$ & 30 & 0.01 & 0.05 & 0.26 \\
\hline Caffeine & Negative & $>100$ & $>100$ & $>100$ & $>100$ & 200 & 11.7 & 0.83 & 66.70 \\
\hline Carisoprodol & Negative & $>100$ & $>100$ & $>100$ & $>100$ & 350 & 23 & 0.40 & 45.05 \\
\hline Cefprozil & Negative & 38.2 & $>100$ & $>100$ & $>100$ & 500 & 24.7 & 0.64 & 70.65 \\
\hline Cefuroxime & Negative & $>100$ & 33 & $>100$ & 9 & 500 & 41.3 & 0.50 & 59.92 \\
\hline Chloramphenicol & Negative & $>100$ & $>100$ & $>100$ & $>100$ & 666 & 10.3 & 0.47 & 69.27 \\
\hline Chlorpropamide & Negative & $>100$ & $>100$ & $>100$ & $>100$ & 500 & 275 & 0.10 & 39.55 \\
\hline Cimetidine & Negative & $>100$ & $>100$ & $>100$ & $>100$ & 1600 & 36.5 & 0.85 & 390.33 \\
\hline Ciprofloxacin & Negative & $>100$ & $>100$ & 73 & $>100$ & 600 & 9.66 & 0.80 & 104.30 \\
\hline Clarithromycin & Negative & $>100$ & $>100$ & 71 & 34.9 & 500 & 5 & 0.41 & 20.32 \\
\hline Clofibrate & Negative & $>100$ & $>100$ & $>100$ & $>100$ & 1000 & 404 & 0.08 & 54.30 \\
\hline Clotrimazole & Negative & 10 & $>100$ & $>100$ & $>100$ & 50 & 0.12 & 0.02 & 0.20 \\
\hline Diltiazem & Negative & $>100$ & $>100$ & $>100$ & $>100$ & 500 & 0.3 & 0.30 & 24.21 \\
\hline Diphenhydramine & Negative & 73 & $>100$ & $>100$ & 43 & 50 & 0.33 & 0.02 & 0.27 \\
\hline Dipyridamole & Negative & 0.8 & $>100$ & $>100$ & 2.8 & 100 & 3.67 & 0.009 & 0.15 \\
\hline Disopyramide & Negative & $>100$ & $>100$ & $>100$ & $>100$ & 800 & 22.4 & 0.50 & 89.75 \\
\hline Entacapone & Negative & 11.4 & 52 & 9 & 0.7 & 200 & 3.28 & 0.02 & 0.94 \\
\hline Erythromycin & Negative & $>100$ & $>100$ & $>100$ & $>100$ & 250 & 1.91 & 0.10 & 2.46 \\
\hline Esomeprasole & Negative & $>100$ & $>100$ & $>100$ & $>100$ & 40 & 7 & 0.03 & 0.44 \\
\hline Famotidine & Negative & $>100$ & $>100$ & $>100$ & $>100$ & 160 & 0.47 & 0.20 & 5.75 \\
\hline Fluoxetine & Negative & $>100$ & 43 & 32 & $>100$ & 80 & 0.98 & 0.06 & 1.09 \\
\hline Furosemide & Negative & $>100$ & $>100$ & $>100$ & 1.7 & 480 & 32.7 & 0.02 & 2.07 \\
\hline Hydroxyzine & Negative & $>100$ & $>100$ & $>100$ & $>100$ & 100 & 0.35 & 0.07 & 1.27 \\
\hline Levofloxacin & Negative & $>100$ & $>100$ & $>100$ & $>100$ & 750 & 25.7 & 0.27 & 44.30 \\
\hline Lopinavir & Negative & 7.4 & $>100$ & 81 & $>100$ & 800 & 18.8 & 0.02 & 2.07 \\
\hline Loracarbef & Negative & $>100$ & $>100$ & $>100$ & $>100$ & 625 & 56.8 & 0.08 & 13.19 \\
\hline Megestrol & Negative & 14.4 & $>100$ & $>100$ & $>100$ & 800 & 2.73 & 0.02 & 3.39 \\
\hline Meloxicam & Negative & 34 & $>100$ & $>100$ & $>100$ & 15 & 9.1 & 0.01 & 0.07 \\
\hline Metformin & Negative & $>100$ & $>100$ & $>100$ & $>100$ & 850 & 9.4 & 1.00 & 448.13 \\
\hline Nabumetone & Negative & $>100$ & $>100$ & $>100$ & 30.3 & 2000 & 315 & 0.01 & 8.99 \\
\hline Nadolol & Negative & 84 & $>100$ & $>100$ & $>100$ & 240 & 2.9 & 0.70 & 38.23 \\
\hline Naproxen & Negative & $>100$ & $>100$ & $>100$ & 25 & 500 & 423 & 0.01 & 5.68 \\
\hline Nifedipine & Negative & 23 & $>100$ & $>100$ & $>100$ & 30 & 0.26 & 0.08 & 0.48 \\
\hline Olanzapine & Negative & $>100$ & $>100$ & $>100$ & $>100$ & 20 & 0.26 & 0.07 & 0.32 \\
\hline Omeprazole & Negative & 22.5 & $>100$ & $>100$ & $>100$ & 40 & 5.41 & 0.05 & 0.66 \\
\hline Paroxetine & Negative & $>100$ & $>100$ & 59.5 & $>100$ & 60 & 0.05 & 0.05 & 0.61 \\
\hline Pentoxyfilline & Negative & $>100$ & $>100$ & 32.9 & $>100$ & 400 & 0.3 & 0.30 & 28.87 \\
\hline Probenecid & Negative & 68.5 & $>100$ & $>100$ & $>100$ & 500 & 122 & 0.25 & 59.70 \\
\hline Propranolol & Negative & $>100$ & $>100$ & $>100$ & $>100$ & 160 & 3.86 & 0.10 & 4.50 \\
\hline Quinidine & Negative & 49.3 & $>100$ & $>100$ & $>100$ & 400 & 4.84 & 0.20 & 17.43 \\
\hline Raloxifene & Negative & 37 & $>100$ & $>100$ & 30.5 & 60 & 0 & 0.05 & 0.42 \\
\hline Ranitidine & Negative & $>100$ & 91 & $>100$ & $>100$ & 150 & 1.5 & 0.15 & 5.00 \\
\hline Rosiglitazone & Negative & 0.6 & $>100$ & $>100$ & 4.3 & 8 & 1.4 & 0.002 & 0.01 \\
\hline Sumatriptan & Negative & $>100$ & $>100$ & $>100$ & $>100$ & 200 & 0.2 & 0.86 & 39.12 \\
\hline Tacrine & Negative & $>100$ & $>100$ & $>100$ & $>100$ & 160 & 0.17 & 0.45 & 24.29 \\
\hline Tetracycline & Negative & $>100$ & $>100$ & $>100$ & $>100$ & 500 & 9 & 0.45 & 37.80 \\
\hline Tramadol & Negative & $>100$ & $>100$ & $>100$ & $>100$ & 100 & 1.01 & 0.80 & 21.06 \\
\hline Trimethobenzamide & Negative & $>100$ & $>100$ & 48.8 & $>100$ & 300 & 10 & 1.00 & 61.49 \\
\hline Valsartan & Negative & 16.2 & $>100$ & $>100$ & $>100$ & 320 & 0.14 & 0.05 & 2.46 \\
\hline Verapamil & Negative & $>100$ & $>100$ & $>100$ & 52.7 & 120 & 0.6 & 0.10 & 1.82 \\
\hline Zolpidem & Negative & 97 & $>100$ & 56 & $>100$ & 10 & 0.39 & 0.08 & 0.205 \\
\hline Acetaminophen & Positive & $>100$ & $>100$ & $>100$ & $>100$ & 1000 & 64.6 & 0.90 & 455.04 \\
\hline Almorexant & Positive & 1.7 & $>100$ & $>100$ & $>100$ & 400 & 0.22 & 0.01 & 0.52 \\
\hline Alpidem & Positive & 9.3 & $>100$ & $>100$ & $>100$ & 50 & 0.07 & 0.01 & 0.05 \\
\hline Amiodarone & Positive & $>100$ & $>100$ & 58 & $>100$ & 600 & 0.7 & 0.04 & 2.51 \\
\hline Amoxicillin*-clavulanate** & Positive & $>100$ & $>100$ & $>100$ & 40 & $300 * / 75 * *$ & $9 * / 7.5 * *$ & $0.88 * / 0.75 * *$ & $56 * / 24 * *$ \\
\hline Azithromycin & Positive & $>100$ & $>100$ & 22.4 & $>100$ & 2000 & 2.36 & 0.93 & 167.75 \\
\hline Benzbromarone & Positive & 4.6 & 1.04 & $>100$ & 1 & 300 & 42.4 & 0.01 & 0.90 \\
\hline Bosentan & Positive & 37 & $>100$ & $>100$ & 22.2 & 125 & 2.54 & 0.02 & 0.35 \\
\hline Bromfenac & Positive & $>100$ & $>100$ & $>100$ & 1 & 50 & 12 & 0.001 & 0.02 \\
\hline Carbamazepine & Positive & $>100$ & $>100$ & $>100$ & $>100$ & 200 & 11.6 & 0.24 & 16.33 \\
\hline Chlormezanone & Positive & $>100$ & $>100$ & $>100$ & $>100$ & 600 & 32.9 & 0.52 & 93.09 \\
\hline Clopidogrel & Positive & 36 & $>100$ & $>100$ & $>100$ & 75 & 0.01 & 0.06 & 0.93 \\
\hline
\end{tabular}


TABLE 1-Continued

\begin{tabular}{|c|c|c|c|c|c|c|c|c|c|}
\hline \multirow{2}{*}{ Compound } & \multirow{2}{*}{$\begin{array}{c}\text { DILI } \\
\text { classification }\end{array}$} & \multicolumn{4}{|c|}{ Estimated $\mathrm{IC}_{50 \mu \mathrm{M}}$} & \multirow{2}{*}{ Dose (mg) } & \multirow{2}{*}{$\mathrm{C}_{\max }(\mu \mathrm{M})$} & \multirow{2}{*}{ fu } & \multirow{2}{*}{$\mathrm{fu}^{*} \mathrm{I}_{\mathrm{in}, \max (\mu \mathrm{M})}$} \\
\hline & & BSEP & MRP2 & MRP3 & MRP4 & & & & \\
\hline Clozapine & Positive & $>100$ & $>100$ & 38 & $>100$ & 300 & 3.67 & 0.03 & 1.95 \\
\hline Cyproterone acetate & Positive & 6.7 & $>100$ & $>100$ & $>100$ & 100 & 0.53 & 0.04 & 0.66 \\
\hline Diclofenac & Positive & $>100$ & $>100$ & $>100$ & 30 & 100 & 0.84 & 0.01 & 0.23 \\
\hline Dilevalol & Positive & $>100$ & $>100$ & $>100$ & $>100$ & 1200 & 1.12 & 0.50 & 122.40 \\
\hline Ethambutol & Positive & $>100$ & $>100$ & 35 & $>100$ & 2500 & 8.4 & 0.80 & 659.32 \\
\hline Felbamate & Positive & $>100$ & $>100$ & $>100$ & $>100$ & 1200 & 192 & 0.78 & 411.68 \\
\hline Flucloxacillin & Positive & 50.1 & $>100$ & $>100$ & $>100$ & 250 & 16 & 0.05 & 2.64 \\
\hline Flutamide & Positive & $>100$ & $>100$ & $>100$ & 10.9 & 250 & 0.36 & 0.06 & 3.64 \\
\hline HCV-796 & Positive & $>100$ & $>100$ & $>100$ & $>100$ & 1000 & 4.93 & 0.48 & 73.26 \\
\hline Isoniazid & Positive & $>100$ & $>100$ & $>100$ & $>100$ & 300 & 26.3 & 1.00 & 172.14 \\
\hline Labetalol & Positive & $>100$ & $>100$ & $>100$ & $>100$ & 600 & 1.36 & 0.50 & 61.66 \\
\hline Lapatanib & Positive & 1.2 & $>100$ & 57.1 & 1.6 & 1250 & 2.12 & 0.01 & 0.92 \\
\hline Lumiracoxib & Positive & $>100$ & $>100$ & $>100$ & 9 & 400 & 25 & 0.02 & 2.32 \\
\hline Methimazole & Positive & 56.1 & $>100$ & $>100$ & $>100$ & 20 & 3.5 & 0.05 & 0.76 \\
\hline Methyldopa & Positive & $>100$ & $>100$ & $>100$ & $>100$ & 500 & 10.4 & 0.90 & 151.40 \\
\hline Metiamide & Positive & $>100$ & $>100$ & 100 & $>100$ & & & & \\
\hline MK-0377 & Positive & $>100$ & $>100$ & 30 & $>100$ & & & & \\
\hline MK-0536 & Positive & $>100$ & $>100$ & $>100$ & $>100$ & 400 & 9.9 & 0.01 & 0.71 \\
\hline MK-0571 & Positive & 1.2 & 8.58 & 17.2 & 0.4 & & & & \\
\hline MK-0633 & Positive & 7.4 & $>100$ & $>100$ & 16.6 & 100 & 4 & 0.08 & 1.49 \\
\hline MK-0773 & Positive & 1.1 & $>100$ & $>100$ & 18.1 & 200 & 31.3 & 0.01 & 0.71 \\
\hline MK-0974 & Positive & 26.3 & $>100$ & $>100$ & 61.4 & 280 & 4 & 0.04 & 1.5 \\
\hline MK-A & Positive & $>100$ & 43 & 82 & $>100$ & 400 & 0.6 & 0.26 & 12.26 \\
\hline MK-2461 & Positive & 4.1 & 48 & $>100$ & 0.7 & 60 & 0.37 & 0.05 & 0.40 \\
\hline MK-2764 & Positive & $>100$ & $>100$ & 33 & $>100$ & 600 & 0.93 & 0.14 & 10.20 \\
\hline MK-3207 & Positive & 0.5 & $>100$ & $>100$ & $>100$ & 900 & 5 & 0.1 & 11.3 \\
\hline MK-3984 & Positive & $>100$ & $>100$ & $>100$ & $>100$ & 125 & 1.8 & 0.03 & 250.05 \\
\hline Nefazodone & Positive & 2.8 & $>100$ & $>100$ & $>100$ & 300 & 6.12 & 0.01 & 0.49 \\
\hline Nelfinavir & Positive & 22.8 & $>100$ & $>100$ & $>100$ & 250 & 5.3 & 0.02 & 0.69 \\
\hline Nevirapine & Positive & 72 & $>100$ & $>100$ & $>100$ & 200 & 5.81 & 0.40 & 22.31 \\
\hline Nitrofurantoin & Positive & $>100$ & $>100$ & $>100$ & 1.3 & 100 & 4.2 & 0.80 & 25.75 \\
\hline Nomifensine & Positive & $>100$ & $>100$ & $>100$ & $>100$ & 200 & 18 & 0.40 & 29.58 \\
\hline Oxandrolone & Positive & $>100$ & $>100$ & 67 & $>100$ & 20 & 1.30 & 0.05 & 0.28 \\
\hline Panadiplon & Positive & $>100$ & $>100$ & $>100$ & $>100$ & & & & \\
\hline Pemoline & Positive & 78 & $>100$ & 55 & $>100$ & 112 & 15.26 & 0.50 & 28.82 \\
\hline Perhexiline maleate & Positive & $>100$ & $>100$ & 30.2 & $>100$ & 200 & 0.24 & 0.10 & 4.83 \\
\hline Ritonavir & Positive & 0.7 & 85 & 31 & 25.5 & 600 & 15.3 & 0.02 & 1.42 \\
\hline Saquinavir & Positive & 11 & $>100$ & 23.9 & $>100$ & 1000 & 8.25 & 0.02 & 2.15 \\
\hline Sitaxsentan & Positive & 4.3 & $>100$ & $>100$ & 13.3 & 100 & 30.6 & 0.01 & 0.45 \\
\hline Sudoxicam & Positive & 33 & $>100$ & $>100$ & 60 & & & & \\
\hline Tasosartan & Positive & 1.7 & $>100$ & $>100$ & 30.8 & 300 & 12.7 & 0.01 & 0.31 \\
\hline Telithromycin & Positive & 4.2 & $>100$ & $>100$ & $>100$ & 400 & 1.02 & 0.40 & 13.54 \\
\hline Ticlopidine & Positive & 73 & $>100$ & $>100$ & $>100$ & 250 & 3.8 & 0.02 & 1.34 \\
\hline Ticrynafen & Positive & 41 & $>100$ & 80.6 & 13 & 500 & 42.3 & 0.02 & 2.14 \\
\hline Tolcapone & Positive & 12.1 & 46 & $>100$ & 0.6 & 200 & 20.9 & 0.001 & 0.07 \\
\hline Troglitazone & Positive & 1.3 & 6.3 & $>100$ & 21.3 & 600 & 7.5 & 0.01 & 0.98 \\
\hline Trovafloxacin mesylate & Positive & $>100$ & $>100$ & $>100$ & $>100$ & 200 & 5 & 0.30 & 11.11 \\
\hline Valproate & Positive & $>100$ & $>100$ & $>100$ & $>100$ & 650 & 477 & 0.19 & 143.83 \\
\hline Verlukast & Positive & 0.9 & 11.1 & 20.1 & 0.8 & 1500 & 241 & 0.001 & 0.44 \\
\hline Ximelagatran & Positive & $>100$ & 38 & $>100$ & $>100$ & & & & \\
\hline Zafirlukast & Positive & 4.5 & 11.5 & $>100$ & 5.8 & 20 & 0.65 & 0.01 & 0.03 \\
\hline Zimelidine & Positive & $>100$ & 58.3 & $>100$ & $>100$ & 600 & 1.64 & 0.09 & 10.99 \\
\hline
\end{tabular}

where accumulation in cells plus bile was determined in MPCCs preincubated in HBSS containing calcium and cellular accumulation of substrate in cells alone was determined in MPCCs preincubated in HBSS buffer devoid of calcium. The $\mathrm{AUC}_{\text {medium }}$ was defined to be a constant (equal to 1), as the concentration of TCA in the incubation media was not expected to change over the course of the experiment (Wolf et al., 2010).

TCA transport was calculated by subtracting the accumulation in stromal cell-only cultures from the accumulation in MPCCs. The data were normalized to percentages of control, where $\mathrm{Cl}_{\text {biliary }}$ or $\mathrm{BEI}$ in the absence of test compound was defined as $100 \%$. $\mathrm{IC}_{50}$ values were obtained by fitting the data using eq. 8 by nonlinear regression analysis using GraphPad Prism:

$$
\operatorname{Control}(\%)=100 /\left((1+\mathrm{I}) / \mathrm{IC}_{50}\right),
$$

where "Control(\%)" represents transporter-mediated uptake/efflux measured in the presence of various concentrations of inhibitor to that in the absence of inhibitor and "I" represents the nominal inhibitor concentration. Compounds were tested up to 25 or $50 \mu \mathrm{M}$ based on solubility limitations. $\mathrm{IC}_{50}$ values were not extrapolated beyond the top test concentration. Data are expressed as means \pm S.E., where S.E. describes the standard error of the curve fit.

Metabolite Identification in MPCCs. After a 24-hour preincubation of test compound in serum-free hepatocyte media with MPCCs, acetonitrile containing $0.1 \%$ formic acid was added to the wells to bring the organic concentration to $75 \%$. Wells were thoroughly mixed, and cell lysate and media were collected in 96-well plates. Plates were then spun at $3000 \mathrm{~g}$ for 5 minutes to precipitate cellular proteins. Supernatant was collected from each well and pooled with $n=3$ replicates to increase metabolite amounts. The metabolite identification was performed using a Xevo G2 Q-TOF high-resolution mass spectrometer (Waters Corp., Milford, MA) equipped with 

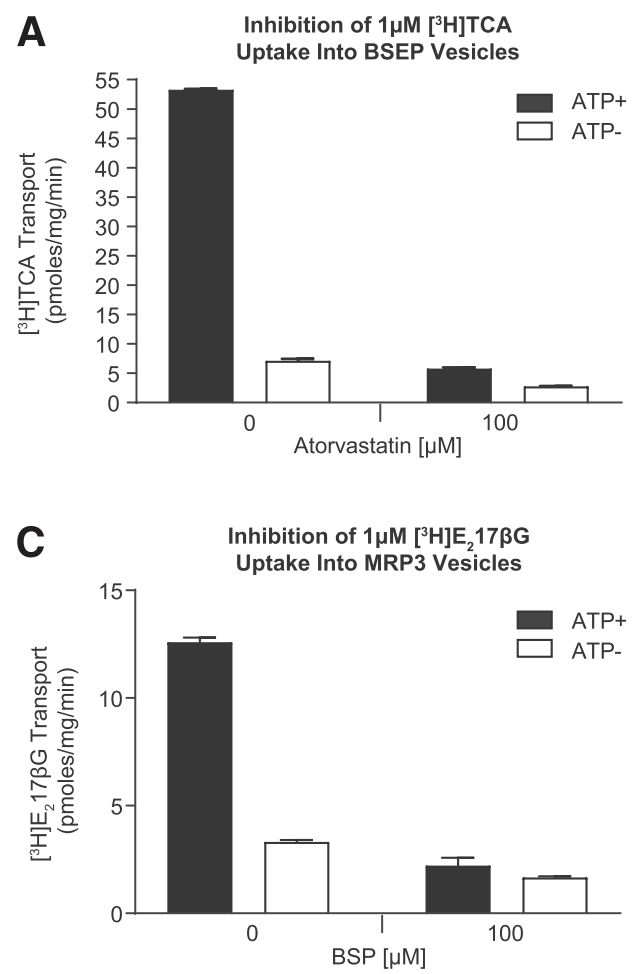
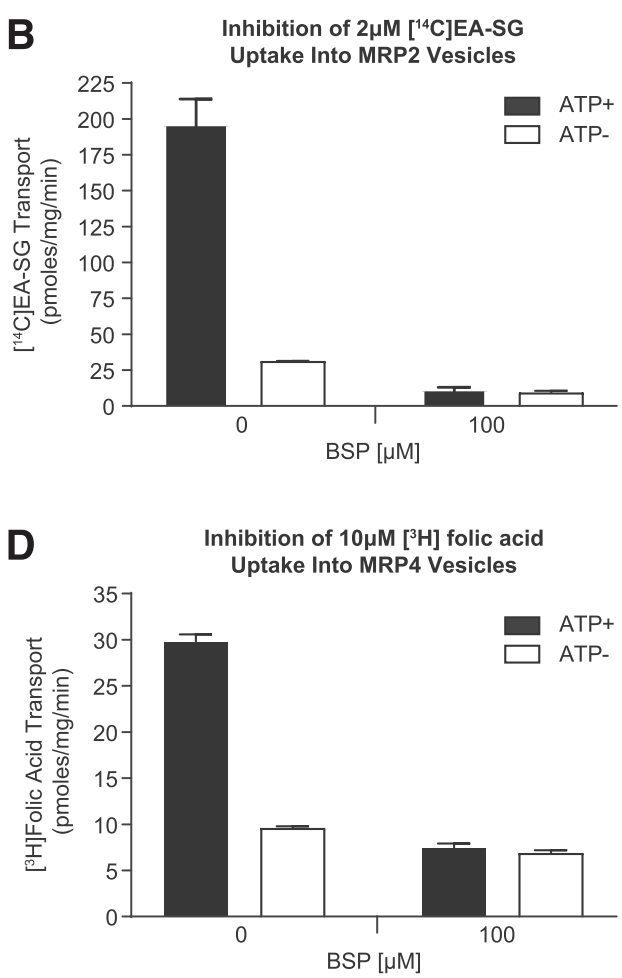

Fig. 2. The inhibitory effect of select inhibitors on the uptake of TCA (A), EA-SG (B), $\mathrm{E}_{2} 17 \beta \mathrm{G}(\mathrm{C})$, and folic acid (D) into membrane vesicles containing BSEP, MRP2, MRP3, and MRP4 in the presence or absence of ATP. $\mathrm{BSP}$, bromosulphophthalein. an electrospray source operated in positive mode and coupled with an Acquity UPLC system (Waters Corp.). Chromatographic separation for metabolite identification was achieved on a Kinetex C18 column $(2.6 \mu, 100 \mathrm{~mm} \times 2.1 \mathrm{~mm}$ internal diameter.; Phenomenex, Torrance, CA). The gradient elution was started from $5 \% \mathrm{~B}$ and increased to $80 \%$ B linearly in 10 minutes, then maintained for 2 minutes and finally decreased to $5 \% \mathrm{~B}$ to equilibrate the column. The flow rate was set at $0.5 \mathrm{ml} / \mathrm{min}$, and the column temperature was maintained at $40^{\circ} \mathrm{C}$. High-resolution mass spectrometry (MS) and tandem MS acquisitions parameters were set as follows: source temperature, $150^{\circ} \mathrm{C}$; desolvation temperature, $350^{\circ} \mathrm{C}$; cone voltage, $35 \mathrm{~V}$. Data were acquired from 80 to $1300 \mathrm{Da}$. The mass spectrometer and UPLC system were operated with MassLynx 4.1 software. The metabolite data processes were done by Metabolynx XS.

\section{Results}

Development of BSEP and MRP2-4 Inhibition Vesicular Screening Assays. Inhibition assays were established to examine the effect of test compound on human BSEP-, MRP2-, MRP3-, and MRP4-mediated transport in membrane vesicles isolated from baculovirus-infected $\mathrm{Sf} 9$ cells containing the transporter of interest. Uptake of probe substrates increased 7.6-, 6.3-, 3.8-, and 3.1-fold in the presence of ATP compared with that observed in the absence of ATP for BSEP, MRP2, MRP3, and MRP4 assays, respectively (Fig. 2). ATP-dependent uptake was abolished by the inhibitors atorvastatin (BSEP) and bromosulphophthalein (MRP2, MRP3, and MRP4), confirming the functionality of the assays. Of note, TCA was not used as the probe substrate in MRP2-4 inhibition assays because assay windows were $<2$-fold (data not shown).
A

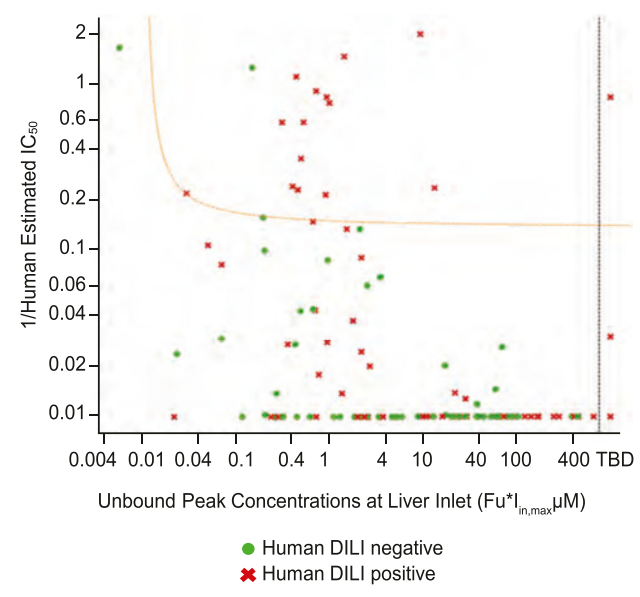

B

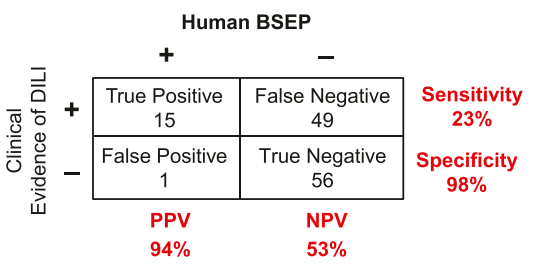

C

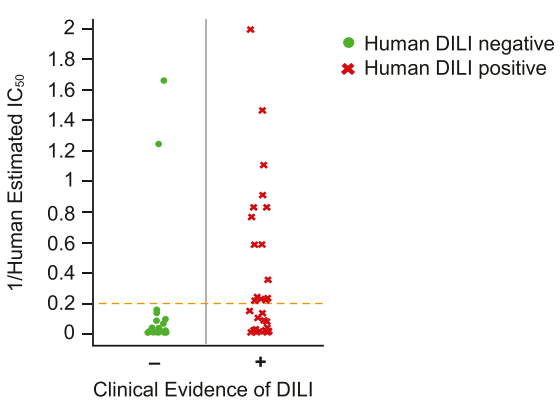

Fig. 3. Establishment of an empirical BSEP cutoff based on a relationship between the estimated BSEP IC $\mathrm{IC}_{50}$ and liver fu* $\mathrm{I}_{\text {in,max }}$ (A, B) The estimated $\mathrm{IC}_{50}$ values for inhibition of BSEP-mediated TCA uptake into membrane vesicles in relation to the $\mathrm{fu}^{*} \mathrm{I}_{\mathrm{in}, \max }$ for DILInegative and -positive compounds (A) with corresponding sensitivity analysis (B). The orange dotted line represents an empirical cutoff (based on eq. 2), where inhibition of BSEP may be predictive of human DILI for compounds that fall above the line. (C) The estimated $\mathrm{IC}_{50}$ values for inhibition of BSEP-mediated TCA uptake into membrane vesicles in relation to DILI categorization. A cutoff of $5 \mu \mathrm{M}$ yields similar specificity as that observed in (A). TBD, to be determined; NPV, negative predictive value; PPV, positive predictive value. 
Establishment of an Empirical Vesicular BSEP Assay Cutoff Based on the Relationship between BSEP IC $_{50}$ and Liver $\mathbf{f u}^{*} \mathbf{I}_{\mathbf{i n} \text {,max }}$. To evaluate the relationship between in vitro BSEP inhibition in membrane vesicles and human DILI risk, the inhibitory effect of 64 DILI-positive and 57 DILI-negative compounds on ATP-dependent $\left[{ }^{3} \mathrm{H}\right] \mathrm{TCA}$ uptake was measured. The resulting estimated $\mathrm{BSEP} \mathrm{IC}_{50}$ values were compared with the compound's fu* $\mathrm{I}_{\text {in,max }}$ (Table 1), which was estimated according to the approach described by Hirano et al. (2006) using the highest clinical dose prescribed to patients and corresponding $\mathrm{C}_{\max }$ values (Fig. 3). An empirical cutoff was then established to limit the number of false positives and to maximize assay specificity. Inhibition of BSEP was predictive of human DILI for compounds that fell above the empirical cutoff, whereas inhibition of BSEP was not predictive of human DILI for compounds below the cutoff. The specificity of the assay was high $(98 \%$, Fig. 3B) with only one false positive (dipyridamole) identified. Sensitivity was low (23\%) as anticipated, most likely because DILI for these compounds was caused by mechanisms other than BSEP inhibition. In the absence of dose and exposure data to calculate fu* $\mathrm{I}_{\mathrm{in}, \max }$, a cutoff of $5 \mu \mathrm{M}$ could be set because, based on a sensitivity analysis, $5 \mu \mathrm{M}$ yielded similar results as

A
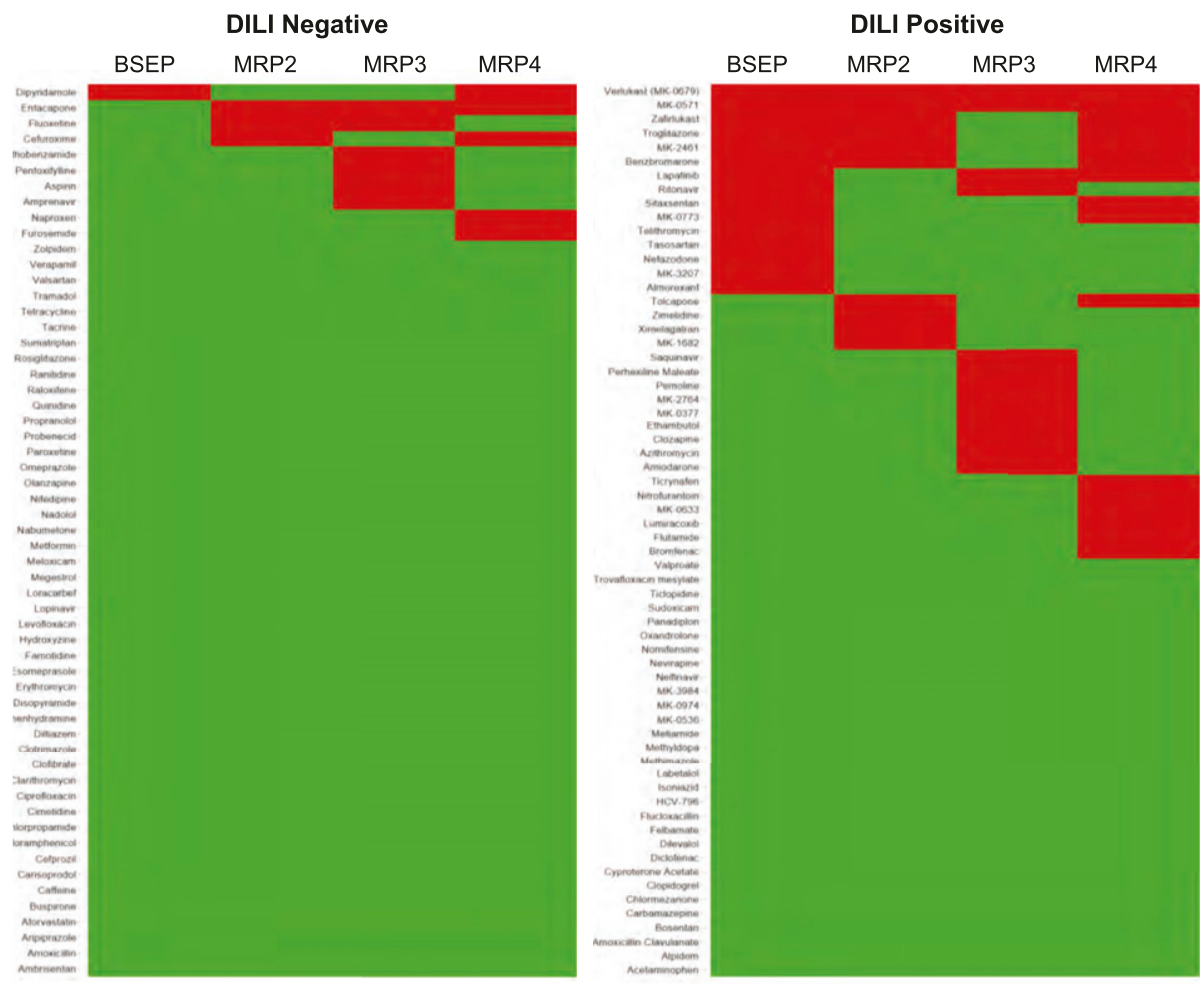

B
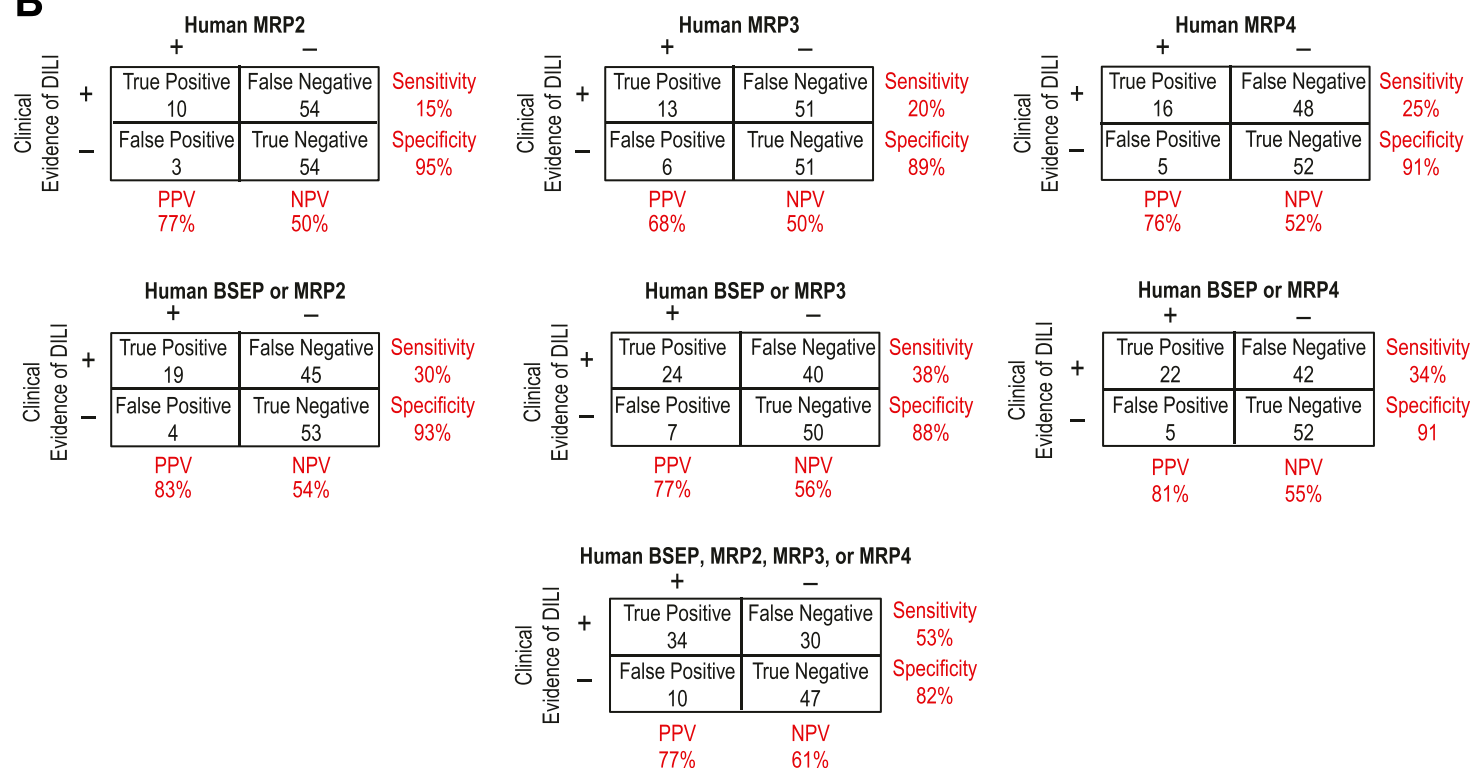

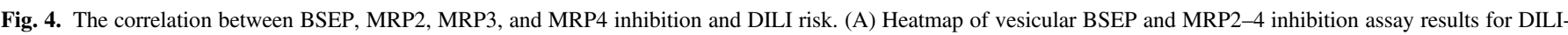

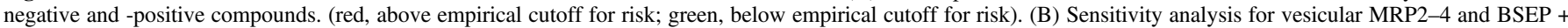
MRP2-4 inhibition assays. NPV, negative predictive value; PPV, positive predictive value. 
the empirical cutoff, with one additional false positive (rosiglitazone) identified (Fig. 3C).

Contribution of Inhibition of MRP2-4 in Addition to BSEP to Predictivity of DILI Risk. To assess whether inhibition of human MRP2, MRP3, and MRP4 would increase the predictivity of human DILI risk, the inhibitory effect of the DILI test set on ATP-dependent uptake of probe substrates was measured in membrane vesicles (Table 1). The results shown in Figure 4 indicated the following: 1) A compound positive for $\mathrm{BSEP}$ inhibition (i.e., $\mathrm{IC}_{50}$ value below the empirical cutoff), regardless of inhibition of MRP2-4, may have an increased DILI risk. 2) Inhibition of MRP2-4 alone lowered the positive predictive value of the assay as compared with BSEP alone because it increased the number of false positives (Fig. 4A, left panel, Fig. 4B). 3) MRP2-4 inhibition in addition to inhibition of BSEP decreased the overall specificity of DILI predictions because it increased the number of false positives (Fig. 4B).

MPCCs Are a Functional Hepatocyte-Based System to Evaluate Inhibition of Bile Salt Efflux. To investigate whether inhibition of BSEP in membrane vesicles correlated with a decrease in the canalicular efflux of TCA in human hepatocytes, MPCCs were explored. Characterization studies were first conducted to determine the localization and activity of BSEP in MPCCs. Figure 5 shows the presence of bile canaliculi as well as the canalicular localization of MRP2 and BSEP by measuring 5-carboxy-2',7' dichlorofluorescein (CDF) accumulation in the canalicular space (Fig. 5A) and the immunolocalization of MRP2 and BSEP using specific antibodies (Fig. 5B), respectively. CDF accumulation in the canalicular space also demonstrated functional MRP2 efflux, as MRP2-mediated efflux of CDF has been shown previously (Zamek-Gliszczynski et al., 2003). Functional activity of BSEP was demonstrated by the time-dependent accumulation of $\left[{ }^{3} \mathrm{H}\right]$ TCA in the presence of calcium (Fig. 6A). This accumulation represents total accumulation of TCA in both the hepatocyte and the bile canalicular space (Wolf et al., 2010) and thus is the sum of sinusoidal uptake and sinusoidal and canalicular efflux. By preincubating cocultures in buffer with or without calcium to modulate cellular tight junctions, distinguishing between TCA accumulation in cellular and bile canalicular compartments was possible (Fig. 6B). This methodology enables the calculation of $\mathrm{Cl}_{\text {biliary }}$ and BEI (reviewed by Swift et al., 2010, Brouwer et al., 2013, and Yang et al., 2016). The $\mathrm{Cl}_{\text {biliary }}$ represents the net effect of uptake of TCA across the sinusoidal membrane into the hepatocyte as well as its efflux across the canalicular membrane into the bile canaliculi. The BEI represents the percent of TCA accumulation that resides in the bile canaliculi (i.e., efflux across the canalicular membrane) and was $>50 \%$ for control incubations in all studies. Inhibition of $\mathrm{Cl}_{\text {biliary }}$ with no change in $\mathrm{BEI}$ indicates that the uptake of TCA is inhibited by the test compound and canalicular efflux of TCA is not, whereas inhibition of both $\mathrm{Cl}_{\text {biliary }}$ and BEI suggests that canalicular efflux of TCA is inhibited with or without concurrent uptake inhibition (Wolf et al., 2010). CsA, a known NTCP, OATP1B, and BSEP inhibitor (Mita et al., 2006; Yoshida et al., 2012), inhibited the $\mathrm{CL}_{\text {biliary }}$ and BEI of TCA after a 10-minute and 24-hour preincubation (Fig. 7; 24-hour data not shown). The resulting $\mathrm{IC}_{50}$ values for CsA inhibition of $\mathrm{CL}_{\text {biliary }}$ of TCA after a 10-minute or 24-hour preincubation were $2.4 \pm 0.8$ and $1.1 \pm 0.2 \mu \mathrm{M}$, respectively. The resulting $\mathrm{IC}_{50}$ values for CsA inhibition of BEI of TCA after a 10-minute or 24-hour preincubation were $4.7 \pm 1.3$ and $2.2 \pm 0.4 \mu \mathrm{M}$, respectively, indicating that both sinusoidal uptake and canalicular efflux were inhibited. Although a 10-minute preincubation with test compound is commonly used (Kenna et al., 2018), a 24-hour preincubation timepoint was included in all studies to allow for potential metabolite effects on BEI to be observed, as metabolite formation is low for the majority of compounds after a 10-minute incubation. In the case of cyclosporin A,

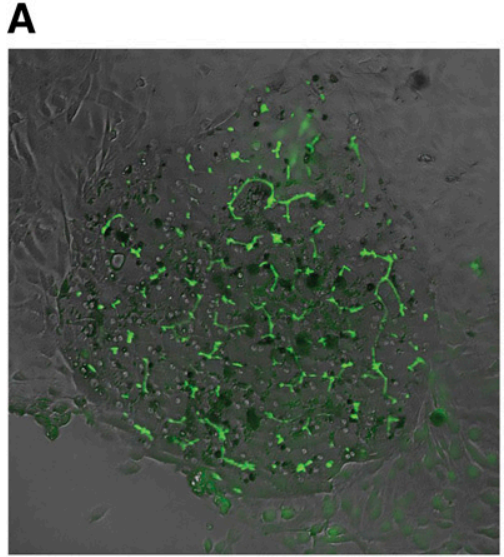

Green $=\operatorname{CDF}(5($ and 6$)$-carboxy-2' 7 'dichlorofluorescein)

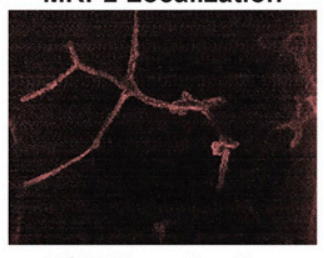

BSEP Localization

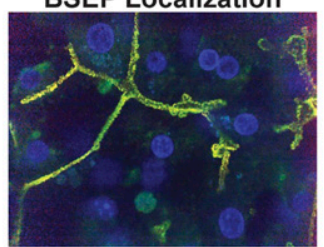

Red $=$ MRP2, Green $=$ BSEP Blue $=$ nuclei
B MRP2 Localization

Fig. 5. Characterization studies show intact bile canaliculi and canalicular membrane localization of MRP2 and BSEP in MPCCs. (A) Accumulation of CDF dye in bile canaliculi of MPCCs. (B) Immunolocalization of MRP2 and BSEP in the canalicular membrane of MPCCs.

the parent accounted for $\sim 95 \%$ of total compound found in the incubation after 24 hours, whereas the remaining $\sim 5 \%$ consisted of two oxidative metabolites and one metabolite formed from demethylation (Supplemental Data). Taken together, these data indicated that MPCCs are a functional hepatocyte co-culture system to examine the effects of test compounds on the uptake and efflux of TCA.

Correlation between Inhibition of BSEP in Membrane Vesicles and Inhibition of BEI in MPCCs. The effect of 19 DILI-positive and 11 DILI-negative compounds on the uptake and efflux of TCA was measured in human MPCCs. The compound set was chosen without bias from the 121 compounds test in membrane vesicles and included both BSEP inhibitors and noninhibitors. The resulting $\mathrm{IC}_{50}$ values for TCA $\mathrm{Cl}_{\text {biliary }}$ and BEI were compared with the fu* $\mathrm{I}_{\mathrm{in}, \max }$ for each compound (Table 2). When the $\mathrm{IC}_{50}$ value was $\leq 10$-fold the fu* $\mathrm{I}_{\text {in,max }}$, a static cutoff used to assess DDI risk for cytochrome P450 enzymes (Food and Drug Administration Center for Drug Evaluation and Research, 2020), compounds were classified as having a risk of inhibiting sinusoidal uptake or both sinusoidal uptake and canalicular efflux in humans. To determine whether BSEP inhibition measured in membrane vesicles correlated with a decrease in TCA canalicular efflux (i.e., a decrease in BEI of TCA) in MPCCs, the $\mathrm{IC}_{50}$ for BEI of TCA after a 24-hour preincubation was compared with the fu* $\mathrm{I}_{\text {in,max }}$ for all compounds tested (Fig. 8A). Nine out of 15 compounds that showed an $\mathrm{IC}_{50}$ value in the BSEP vesicular inhibition assay of $<5 \mu \mathrm{M}$ did not demonstrate an $\mathrm{IC}_{50}$ for BEI that was less than 10 -fold the fu* $\mathrm{I}_{\text {in,max, }}$ (Fig. $8 \mathrm{~B}$ ), suggesting that BSEP inhibition in humans would be unlikely at clinically relevant exposures. A sensitivity analysis (Fig. 8C) showed that the specificity of the MPCC assay to predict DILI-positive compounds using the calculated BEI in conjunction with the $\mathrm{fu}^{*} \mathrm{I}_{\text {in,max }}$ was high (89\%), with only one false positive (lopinavir) identified. Interpretation of the false positive is confounded by the fact that lopinavir is coadministered with ritonavir as a pharmacokinetic enhancer in the form of Kaletra and some degree of serum aminotransferase elevations occur in a high proportion of patients taking lopinavir containing antiretroviral regimens (livertox. nih.gov).

Case Study: Effect of the GPR40 Agonist MK-8666 on Bile Salt Transport Inhibition. MK-8666, a small molecule agonist of the GPR40 receptor that was in development for the treatment of type 2 diabetes mellitus (Hyde et al., 2016), was terminated due to concerns about its liver safety. As detailed in Shang et al. (2020), "a 53-year-old 
A

Accumulation of $1 \mu \mathrm{M}\left[^{3} \mathrm{H}\right] \mathrm{TCA}$

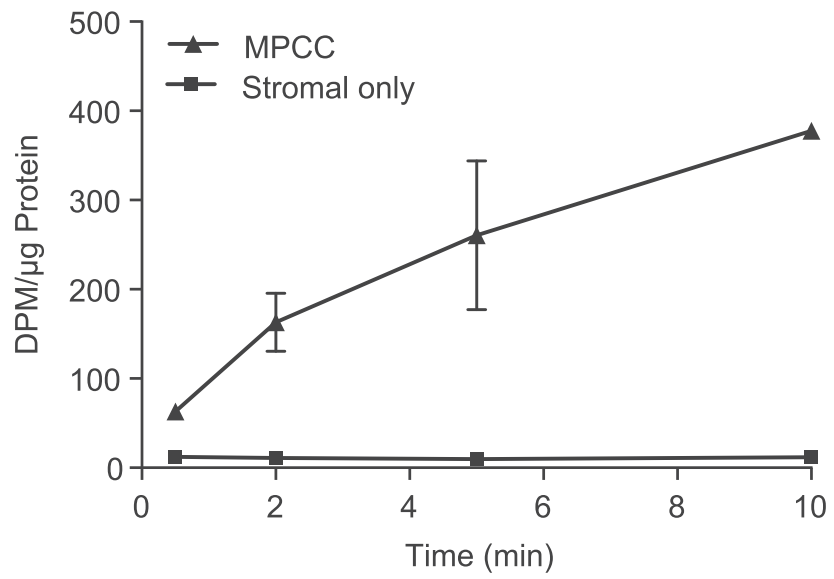

B

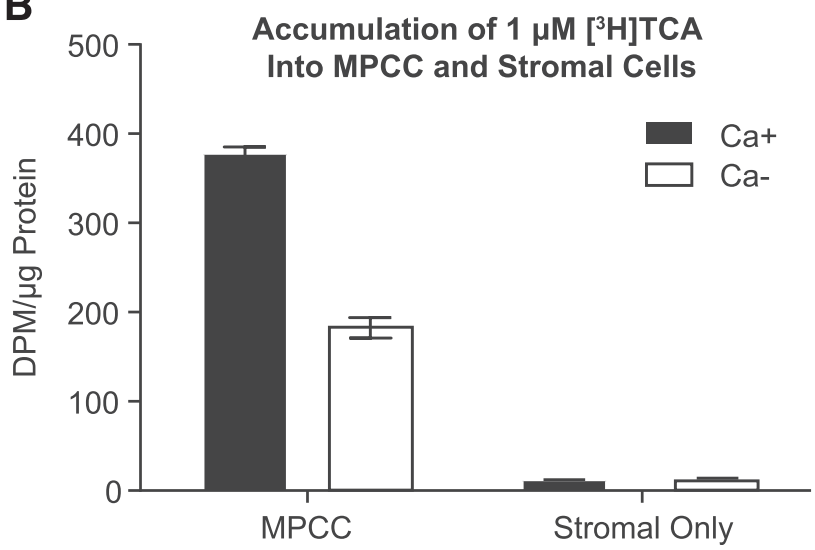

Fig. 6. Accumulation of TCA into MPCCs. (A) Time course of TCA accumulation into MPCCs and stromal cells in the presence of calcium. (B) Uptake and efflux of TCA into MPCCs and stromal cells in the presence and absence of calcium at 10 minutes.DPM, disintegrations per minute.

male subject in the 150-mg QD cohort demonstrated increases in alanine aminotransferase (ALT) and aspartate aminotransferase (AST) above baseline prior to dosing on day 3 and showed an upward trend on continued dosing. The highest observed ALT and AST values were measured $24 \mathrm{~h}$ after the final dose of MK-8666 $(4-5 \times$ and $2-3 \times$ upper-limit-of-normal, respectively), along with concurrent elevation in alkaline phosphatase $(1.4 \times$ upper-limit-of-normal). These findings reversed within 2 weeks after the completion of dosing to normal or nearnormal concentrations. Our company terminated the clinical development of MK-8666 in anticipation of an unfavorable benefit/risk profile" .

To assess whether inhibition of BSEP could contribute to the liver toxicity observed for MK-8666, the $\mathrm{IC}_{50}$ for $\mathrm{BSEP}$ was measured in membrane vesicles: the $\mathrm{IC}_{50}$ was found to be $0.8 \pm 0.08 \mu \mathrm{M}$ (Fig. 9A), which was above the empirical cutoff established in Figure 3A. Subsequently, the inhibition of the uptake and efflux of TCA by MK-8666 in MPCCs was determined. After a 24-hour preincubation, MK-8666 inhibited the $\mathrm{Cl}_{\text {biliary }}$ of TCA with an $\mathrm{IC}_{50}$ of $23.6 \pm 10.5 \mu \mathrm{M}$ (data not shown). At 25 and $50 \mu \mathrm{M}$, the highest concentrations tested, MK-8666 inhibited $\sim 31 \%$ and $97 \%$ of the BEI of $\left[{ }^{3} \mathrm{H}\right] \mathrm{TCA}$, respectively, resulting in an $\mathrm{IC}_{50}$ of $>25 \mu \mathrm{M}$ (Fig. 9B). The $\mathrm{IC}_{50}$ values were $>10$-fold the calculated fu* $\mathrm{I}_{\mathrm{in} \text {,max }}$ even at a dose of $500 \mathrm{mg}$.

To explore whether metabolism of MK-8666 in MPCCs explains the discrepancy between potency in the vesicular and MPCC inhibition assays, the metabolism of MK-8666 was examined. After a 24-hour incubation, approximately $80 \%$ of MK-8666 was converted to an acylglucuronide (AG) metabolite (Fig. 9C). Follow-up studies in BSEP membrane vesicles showed that the MK-8666-AG was not an inhibitor of BSEP-mediated $\left[{ }^{3} \mathrm{H}\right] \mathrm{TCA}$ uptake $\left(\mathrm{IC}_{50}>25 \mu \mathrm{M}\right.$ ) (Fig. 9D).

\section{Discussion}

In this study, we sought to understand the relationship between in vitro BSEP inhibition measured in membrane vesicles and human DILI risk by examining the inhibition potential of 121 DILI-positive or -negative compounds. Through the establishment of an empirical cutoff designed to maximize assay specificity, a relationship was established between vesicular BSEP inhibition and DILI risk by comparing BSEP inhibition potency with the calculated fu* $\mathrm{I}_{\text {in,max }}$. Although inhibition of BSEP in the vesicular inhibition assay may be predictive of DILI risk for compounds above the empirical cutoff, causality has not been demonstrated.

Although membrane vesicles can be used as a first step to assess BSEP inhibition, they lack metabolic activity and capture only one factor involved in bile acid disposition (Kenna et al., 2018). As such, false positives and negatives would be expected based on the vesicle assay alone. MPCCs, in contrast, allow for a more holistic assessment of bile salt transport inhibition, as multiple transport and metabolic pathways are active (Khetani and Bhatia, 2008; (Ramsden et al., 2014); Moore et al., 2016; Kratochwil et al., 2018). To test the hypothesis that BSEP inhibition in vesicles results in decreased BEI in MPCCs, studies with a subset of compounds were conducted in MPCCs to assess the inhibition of both uptake and efflux of TCA.
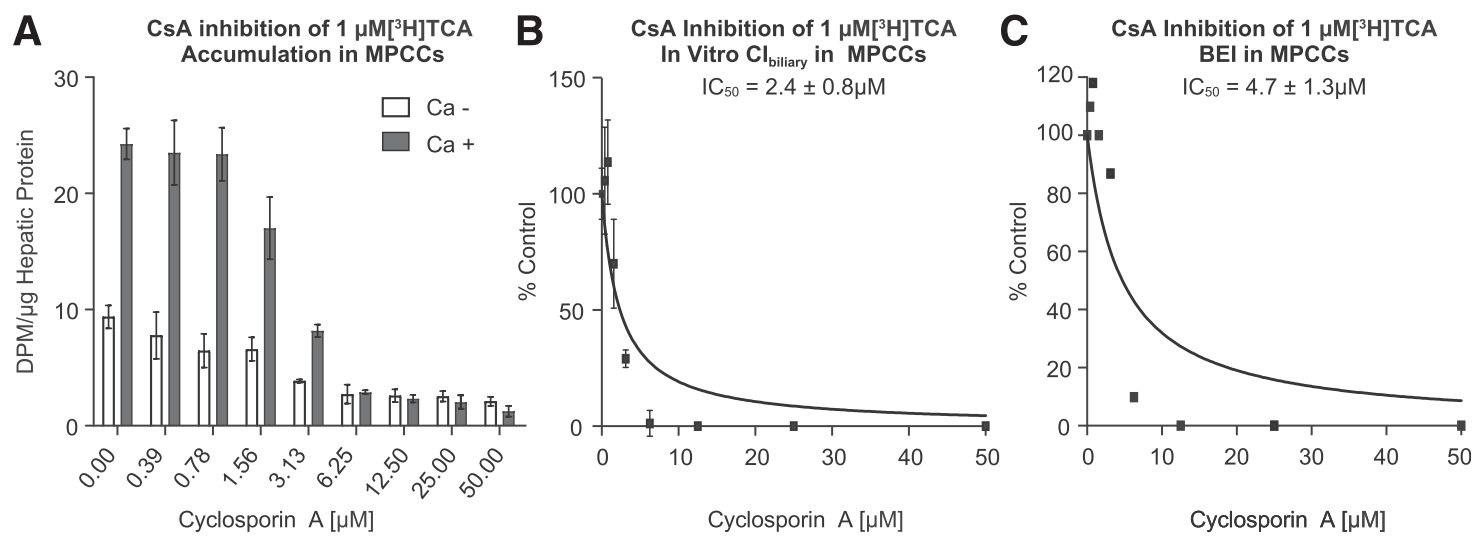

Fig. 7. Inhibition of $\mathrm{Cl}_{\text {biliary }}$ and BEI of TCA in MPCCs by CsA after a 10-minute pretreatment. (A) The inhibitory effect of CsA on the accumulation of $1 \mu \mathrm{M}\left[{ }^{3} \mathrm{H}\right] \mathrm{TCA}$ in the presence or absence of calcium in MPCCs. (B) The inhibitory effect of CsA on $\mathrm{Cl}_{\text {biliary }}$ in human MPCCs. (C) The inhibitory effect of CsA on BEI in MPCCs. Results from a typical experiment are shown. Similar results were observed in five independent experiments.DPM, disintegrations per minute. 
TABLE 2

$\mathrm{IC}_{50}$ values for $\mathrm{Cl}_{\text {biliary }}$ and BEI of TCA generated in MPCCs for DILI-negative and -positive compounds

Bold values indicated values within 10 -fold of the calculated fu* $\mathrm{I}_{\mathrm{in} \text {,max }}$. Metabolite identification high-resolution MS data included as Supplemental Data

\begin{tabular}{|c|c|c|c|c|c|c|c|c|}
\hline \multirow{3}{*}{ Compound } & \multirow{3}{*}{$\begin{array}{c}\text { DILI } \\
\text { classification }\end{array}$} & \multicolumn{4}{|c|}{ MPCCs } & \multirow{3}{*}{ Vesicles BSEP $\mathrm{IC}_{50(\mu \mathrm{M})}$} & \multirow{3}{*}{$\mathrm{fu}^{*} \mathrm{I}_{\mathrm{in}, \max (\mu \mathrm{M})}$} & \multirow{3}{*}{ Met ID HR/MS summary } \\
\hline & & \multicolumn{2}{|c|}{ in vitro $\mathrm{Cl}_{\text {biliary }} \mathrm{IC}_{50}(\mu \mathrm{M})$} & \multicolumn{2}{|c|}{$\mathrm{BEI} \mathrm{IC}_{50(\mu \mathrm{M})}$} & & & \\
\hline & & $10 \mathrm{~min}$ & $24 \mathrm{~h}$ & $10 \mathrm{~min}$ & $24 \mathrm{~h}$ & & & \\
\hline Ambrisantan & Negative & $>50$ & $30.7 \pm 14.1$ & $>50$ & $23.6 \pm 4$ & $>100$ & 0.12 & $\begin{array}{c}\text { Parent } \sim 23 \% \\
\text { four metabolites }\end{array}$ \\
\hline Atorvastatin & Negative & $>50$ & $12.9 \pm 3.1$ & $>50$ & $34.6 \pm 4.8$ & 6.3 & 0.19 & $\begin{array}{c}\text { Parent } \sim 65 \% \\
\text { five metabolites }\end{array}$ \\
\hline Buspirone & Negative & $>50$ & $>50$ & $>50$ & $>50$ & $>100$ & 0.26 & $\begin{array}{c}\text { Parent } 72 \% \\
\text { five metabolites }\end{array}$ \\
\hline Dipyridamole & Negative & $19.6 \pm 7.8$ & $19.5 \pm 7.5$ & $33.2 \pm 14$ & $>25$ & 0.8 & 0.15 & $\begin{array}{c}\text { Parent } \sim 0 \% \\
\text { one metabolite }\end{array}$ \\
\hline Entacapone & Negative & $>50$ & $42.8 \pm 8.9$ & $>50$ & $>50$ & 11.4 & 0.94 & $\begin{array}{c}\text { Parent } \sim 0 \% \\
\text { four metabolites }\end{array}$ \\
\hline Lopinavir & Negative & $2.8 \pm 0.6$ & $3.2 \pm 0.9$ & $18.9 \pm 3.5$ & $9.8 \pm 1.9$ & 7.4 & 2.07 & $\begin{array}{c}\text { Parent } 78 \% \\
\text { five metabolites }\end{array}$ \\
\hline Metformin & Negative & $>50$ & $>50$ & $>50$ & $>50$ & $>100$ & 448.13 & Parent $\sim 100 \%$ \\
\hline Pioglitazone $^{a}$ & Negative & $4.9 \pm 1.1$ & $0.9 \pm 0.3$ & $24.8 \pm 6.6$ & $11.6 \pm 3.4$ & $<1$ & 0.13 & $\begin{array}{l}\text { Parent } \sim 84 \% \\
11 \text { metabolite }\end{array}$ \\
\hline Quinidine & Negative & $>50$ & $>50$ & $>50$ & $>50$ & 49.3 & 17.43 & $\begin{array}{l}\text { Parent } \sim 87 \% \\
\text { four metabolites }\end{array}$ \\
\hline Rosiglitazone & Negative & $2.9 \pm 1.8$ & $2.6 \pm 0.7$ & $23.2 \pm 8.1$ & $>50$ & 0.6 & 0.01 & $\begin{array}{l}\text { Parent } \sim 91 \% \\
\text { one metabolite }\end{array}$ \\
\hline Valsartan & Negative & $>50$ & $>50$ & $>50$ & $>50$ & 16.2 & 2.46 & Parent $\sim 100 \%$ \\
\hline Acetaminophen & Positive & $>50$ & $>50$ & $>50$ & $>50$ & $>100$ & 455.04 & $\begin{array}{l}\text { Parent } \sim 97 \% \\
\text { one metabolite }\end{array}$ \\
\hline Almorexant & Positive & $53.3 \pm 22.3$ & $28.3 \pm 17.6$ & $>50$ & $>50$ & 1.7 & 0.52 & $\begin{array}{l}\text { Parent } \sim 90 \% \\
\text { eight metabolites }\end{array}$ \\
\hline Benzbromarone & Positive & $3.7 \pm 0.7$ & $2.5 \pm 1.1$ & $31.7 \pm 7.9$ & $5.4 \pm 1$ & 4.6 & 0.9 & $\begin{array}{c}\text { Parent } \sim 90 \% \\
\text { three metabolites }\end{array}$ \\
\hline Bosentan & Positive & $38.3 \pm 9.5$ & $10.9 \pm 2.7$ & $>50$ & $37 \pm 5.9$ & 37 & 0.35 & $\begin{array}{l}\text { Parent } \sim 87 \% \\
\text { four metabolites }\end{array}$ \\
\hline Cyclosporin A & Positive & $2.4 \pm 0.8$ & $1.1 \pm 0.2$ & $4.7 \pm 1.3$ & $2.2 \pm 0.4$ & 0.3 & 4.7 & $\begin{array}{c}\text { Parent } \sim 95 \% \\
\text { three metabolites }\end{array}$ \\
\hline Cyproterone acetate & Positive & $26.5 \pm 7.9$ & $17.6 \pm 8.6$ & $>50$ & $>50$ & 6.7 & 0.66 & $\begin{array}{c}\text { Parent } 59 \% \\
\text { three metabolites }\end{array}$ \\
\hline Lapatanib & Positive & $>25$ & $>25$ & $>25$ & $>25$ & 1.2 & 0.92 & $\begin{array}{l}\text { Parent } \sim 61 \% \\
\text { three metabolites }\end{array}$ \\
\hline MK-0773 & Positive & $27.2 \pm 11.2$ & $15.5 \pm 2.3$ & $>50$ & $>50$ & 1.1 & 0.71 & $\begin{array}{l}\text { Parent } \sim 88 \% \\
\text { four metabolites }\end{array}$ \\
\hline MK-0974 & Positive & $15.3 \pm 5$ & $11.7 \pm 5$ & $46 \pm 17.6$ & $16 \pm 8.1$ & 26.3 & 1.5 & $\begin{array}{l}\text { Parent } \sim 96 \% \\
\text { three metabolites }\end{array}$ \\
\hline MK-3207 & Positive & $43 \pm 9.9$ & $>50$ & $>50$ & $>50$ & 0.5 & 11.3 & $\begin{array}{l}\text { Parent } \sim 91 \% \\
\text { two metabolites }\end{array}$ \\
\hline Nefazodone & Positive & $>50$ & $30.9 \pm 9.3$ & $>50$ & $>50$ & 2.8 & 0.49 & $\begin{array}{l}\text { Parent } \sim 80 \% \\
\text { six metabolites }\end{array}$ \\
\hline Ritonavir & Positive & $6.9 \pm 2.4$ & $4.1 \pm 1.6$ & $38.5 \pm 7.6$ & $6.9 \pm 2.1$ & 0.7 & 1.42 & $\begin{array}{c}\text { Parent } 99 \% \\
\text { one metabolite }\end{array}$ \\
\hline Sitaxsentan & Positive & $6.5 \pm 2.1$ & $3.3 \pm 0.9$ & $>50$ & $11.9 \pm 2.2$ & 4.3 & 0.45 & $\begin{array}{l}\text { Parent } \sim 99 \% \\
\text { one metabolite }\end{array}$ \\
\hline Tasosartan & Positive & $44.3 \pm 16.2$ & $9.7 \pm 2.9$ & $>50$ & $39 \pm 8.4$ & 1.7 & 0.31 & $\begin{array}{l}\text { Parent } \sim 75 \% \\
\text { six metabolites }\end{array}$ \\
\hline Telithromycin & Positive & $39.6 \pm 17.2$ & $21.7 \pm 11.7$ & $>50$ & $39.8 \pm 7.7$ & 4.2 & 13.54 & $\begin{array}{l}\text { Parent } \sim 79 \% \\
\text { four metabolites }\end{array}$ \\
\hline Tolcapone & Positive & $20.2 \pm 6.4$ & $14.5 \pm 2$ & $>50$ & $>50$ & 12.1 & 0.07 & $\begin{array}{c}\text { Parent } \sim 4 \% \\
\text { two metabolites }\end{array}$ \\
\hline Troglitazone & Positive & $2.2 \pm 0.3$ & $0.8 \pm 0.2$ & $14.3 \pm 2.9$ & $8.5 \pm 1.7$ & 1.3 & 0.98 & $\begin{array}{c}\text { Parent } \sim 1 \% \\
\text { six metabolites }\end{array}$ \\
\hline Verlukast & Positive & $15.2 \pm 3.5$ & $12.1 \pm 2.9$ & $>50$ & $>50$ & 0.9 & 0.44 & $\begin{array}{c}\text { Parent } \sim 87 \% \\
\text { three metabolites }\end{array}$ \\
\hline Zafirlukast & Positive & $11.7 \pm 4.5$ & $3 \pm 0.7$ & $>50$ & $8.4 \pm 1.5$ & 4.5 & 0.03 & $\begin{array}{c}\text { Parent } \sim 71 \% \\
\text { three metabolites }\end{array}$ \\
\hline
\end{tabular}

${ }^{a}$ Pioglitazone was not included in original test set. Pioglitazone fu* $\mathrm{I}_{\mathrm{in}, \max }$ based on a dose of $45 \mathrm{mg}$ with corresponding $\mathrm{C}_{\max }$ of $4.2 \mu \mathrm{M}$ and an unbound fraction of 0.01 . HR/MS, high-resolution MS.

Inhibition of BSEP in vesicles was not predictive of inhibition of canalicular efflux of TCA in MPCCs for some compounds, including the antithrombotic dipyridamole, the false positive in the vesicular analysis (Figs. 3A and 8B). Overall, the data show that there is no correlation between the $\mathrm{IC}_{50}$ values generated in the two assays (Table 2).
Approximately $13 \%$ of the 121 compounds tested in the vesicular inhibition test set warranted further evaluation in MPCCs based on the cutoff of $5 \mu \mathrm{M}$. Of those tested in MPCCs, $33 \%$ were found to have the potential to inhibit canalicular efflux of bile salts in vivo. As an example, metabolism of dipyridamole in MPCCs likely explains the discrepancy 
A - Human DILI negative $\quad *$ Human DILI positive

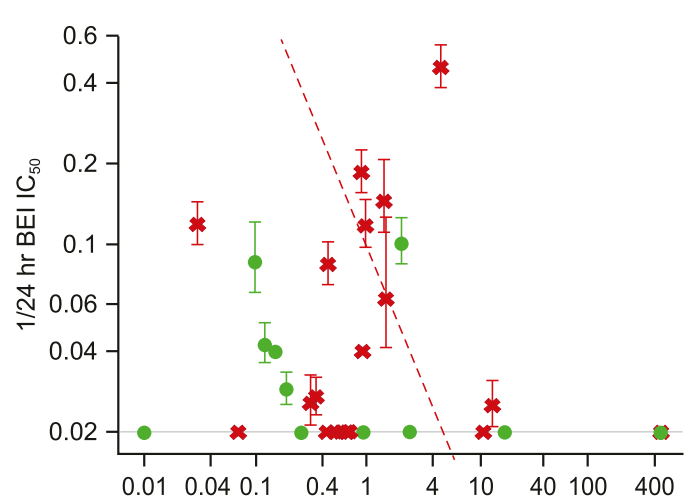

Unbound Peak Concentrations at Liver Inlet $\left(\mathrm{Fu}^{*} \mathrm{I}_{\mathrm{in}, \max } \mu \mathrm{M}\right)$

C

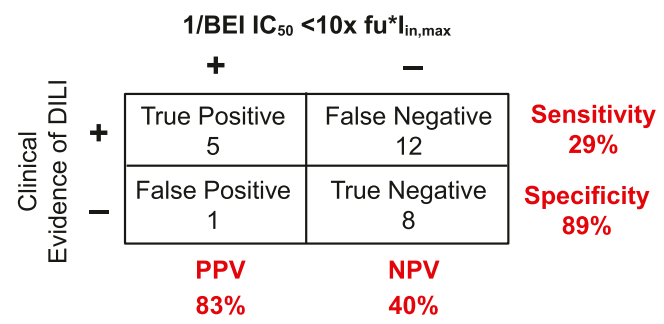

B

\begin{tabular}{|l|l|l|}
\hline \multicolumn{1}{|c|}{ Compound } & $\begin{array}{c}\text { BSEP } \\
\text { Vesicular } \\
\text { Inhibition }\end{array}$ & $\begin{array}{c}\text { MPCC BEI } \\
\text { Inhibition }\end{array}$ \\
\hline Dipyridamole & & \\
\hline Lopinavir & & \\
\hline Ambrisantan & & \\
\hline Atorvastatin & & \\
\hline Buspirone & & \\
\hline Entacapone & & \\
\hline Pioglitazone & & \\
\hline Rosiglitazone & & \\
\hline Valsartan & & \\
\hline Metformin & & \\
\hline Quinidine & & \\
\hline
\end{tabular}

DILI Positive

\begin{tabular}{|l|l|l|}
\hline Compound & $\begin{array}{c}\text { BSEP } \\
\text { Vesicular } \\
\text { Inhibition }\end{array}$ & $\begin{array}{c}\text { MPCC BEI } \\
\text { Inhibition }\end{array}$ \\
\hline Benzbromarone & & \\
\hline Cyclosporine A & & \\
\hline Ritonavir & & \\
\hline Telithromycin & & \\
\hline Troglitazone & & \\
\hline Almorexant & & \\
\hline Lapatanib & & \\
\hline MK-0773 & & \\
\hline Nefazodone & & \\
\hline Sitaxsentan & & \\
\hline Tasosartan & & \\
\hline Verlukast & & \\
\hline Zafirflukast & & \\
\hline MK-3207 & & \\
\hline Bosentan & & \\
\hline Cyproterone & & \\
\hline MK-0974 & & \\
\hline Tolcapone & & \\
\hline Acetaminophen & & \\
\hline
\end{tabular}

Fig. 8. Establishment of a cutoff in MPCCs to assess BSEP inhibition potential. (A) IC $\mathrm{C}_{50}$ values for the BEI of TCA in relation to the fu* $\mathrm{I}_{\text {in,max }}$ for DILI-negative and -positive compounds. (B) Correlation between BSEP vesicular and MPCC BEI inhibition assay results (red, assay positive; green, assay negative; gray, inconclusive due to solubility limitations). (C) Corresponding sensitivity analysis for (A). The red dotted line in (A) represents a 10-fold difference between $1 / \mathrm{IC}_{50}$ and fu* $\mathrm{I}_{\mathrm{in}, \mathrm{max}}$. The black solid line represents cases in which the $\mathrm{IC}_{50}$ was more than the highest concentration tested. Since the $\mathrm{IC}_{50}$ is unknown, it cannot be compared with the fu* $\mathrm{I}_{\mathrm{inn}, \mathrm{max}}$ for compounds that fall to the right of the red dotted line and thus is not included in the sensitivity analysis in (B). NPV, negative predictive value; PPV, positive predictive value.

between potency in the vesicular and MPCC inhibition assays, as dipyridamole was completely converted to an AG metabolite in the incubation (Supplemental Data). In addition to metabolism, discrepancies between inhibition observed in vesicles and MPCCs may be explained by protein binding, intracellular sequestration, and/or compensatory mechanisms such as potential activation of various (nuclear) hormone receptors. Thus, caution is warranted in the interpretation of vesicular inhibition data alone. Importantly, our analysis in MPCCs does not consider the type of DILI associated with a compound but rather focuses on potential to inhibit biliary efflux. Since inhibition of biliary efflux is only one potential mechanism causing/correlating with cholestatic liver injury ((Balistreri et al., 2005); Burbank et al., 2016; Deferm et al., 2019), compounds known to be cholestatic in humans, such as troglitazone, nefazodone, and bosentan, may or may not be flagged using a 10-fold cutoff between the TCA BEI IC ${ }_{50}$ and fu* $\mathrm{I}_{\mathrm{in} \text {,max }}$ indicating altered transporter-mediated efflux.
Several others have examined the relationship between in vitro BSEP inhibition and prediction of human DILI risk (Wolf et al., 2010; (Morgan et al., 2010), 2013; Dawson et al., 2012; Pedersen et al., 2013; Yucha et al., 2017). Although these studies have linked BSEP inhibition to DILI risk, most relationships have been built on BSEP potency alone or comparisons of BSEP potency and total (bound plus unbound) drug concentrations. However, based on the free drug hypothesis, correlations between total drug exposure and BSEP inhibition are unlikely to be causal. The most relevant exposure parameter with which to compare BSEP potency is the unbound intrahepatic concentration of the drug. As this is difficult to measure in vivo (Guo et al., 2018), we compared BSEP potency with $\mathrm{fu}^{*} \mathrm{I}_{\mathrm{in,max}}$. This value can serve as a surrogate for unbound intracellular levels for nontransporter substrates, as unbound plasma and tissue concentrations should be in equilibrium at steady state. Of note, most compounds showing discrepancies between assays were not known to be substrates for uptake transporters. 

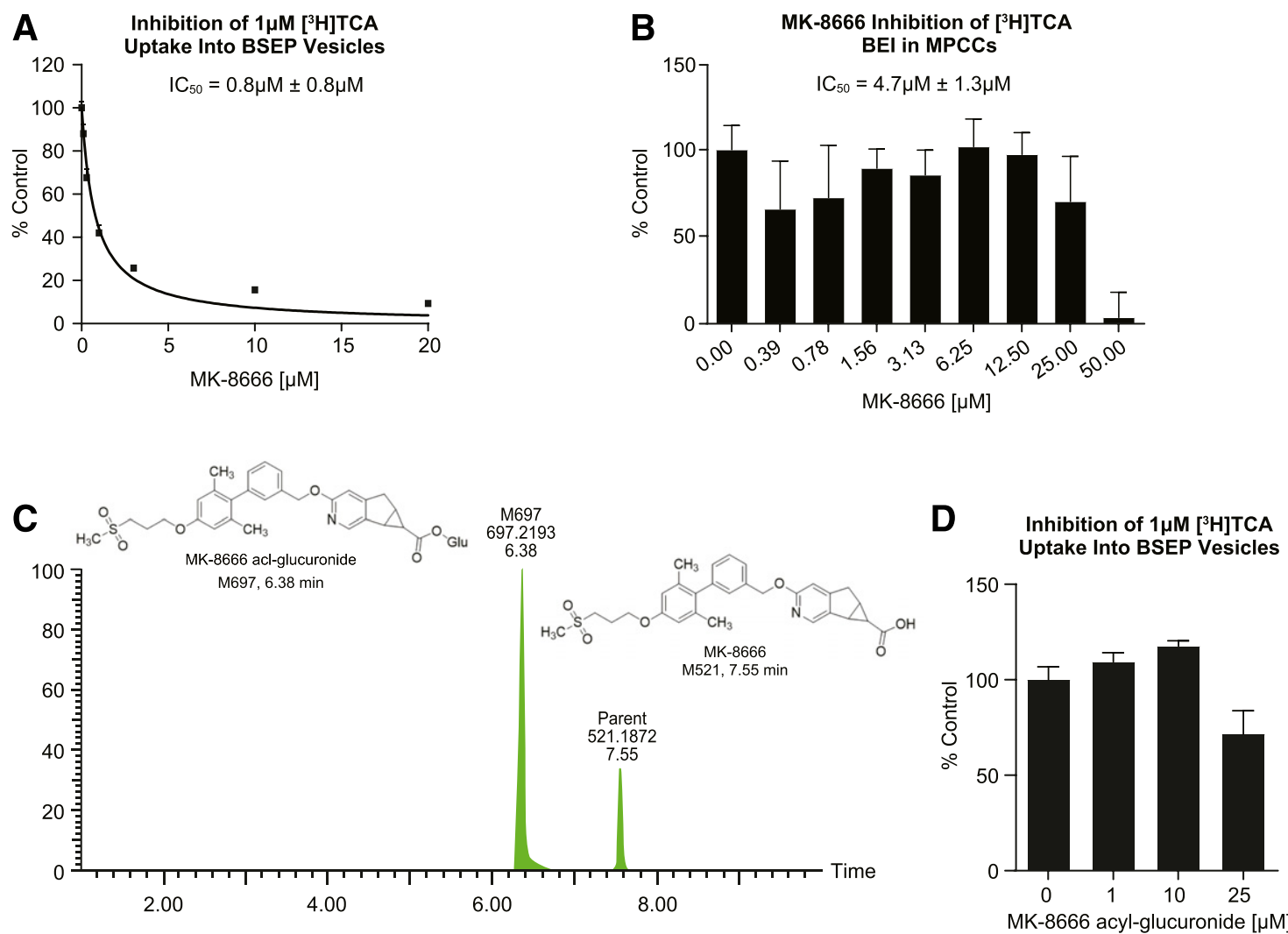

Fig. 9. Inhibition profile of MK-8666 and MK-8666 acyl glucuronide. (A) MK-8666 inhibition of BSEP-mediated $1 \mu \mathrm{M}\left[{ }^{3} \mathrm{H}\right] \mathrm{TCA}$ uptake (\% control) in membrane vesicles (B) The inhibitory effect of MK-8666 on BEI in MPCCs after a 24-hour preincubation. (C) High-resolution MS analysis of MK-8666 MPCC samples after a 24 -hour preincubation. (D) MK-8666 acyl-glucuronide inhibition of BSEP-mediated $1 \mu \mathrm{M}\left[{ }^{3} \mathrm{H}\right] \mathrm{TCA}$ uptake (\% control) in membrane vesicles.

In a recently published white paper, the evaluation of BSEP inhibition was recommended in drug discovery and development to aid in DILI risk assessment (Kenna et al., 2018). A proposed guided workflow included initial screening in membrane vesicles and utilization of a $25 \mu \mathrm{M}$ static cutoff followed by comparison of the $\mathrm{BSEP} \mathrm{IC}_{50}$ to total steady-state $\mathrm{C}_{\max }\left(\mathrm{C}_{\max , \mathrm{ss}}\right)$. In this analysis, a margin of $<10$ (i.e., $\mathrm{BSEP} \mathrm{IC}_{50}<10$-fold total $\mathrm{C}_{\mathrm{max}, \mathrm{ss}}$ ) was proposed to suggest a potential risk, based largely on the data published by Morgan et al. (2010). In the present study, this cutoff resulted in an increased rate of false positives (data not shown). Our BSEP vesicular analysis offers improvement to previous analyses, as the use of $\mathrm{fu}^{*} \mathrm{I}_{\mathrm{in}, \max }$ is a more relevant exposure parameter that increases assay specificity. In addition, our analysis also suggests the use of $5 \mu \mathrm{M}$ as a static cutoff when dose and $\mathrm{C}_{\max }$ predictions are not available, allowing for early assessment of risk in drug discovery. It should be noted that the results are dependent on the selected test set and the extent to which the test set is representative of the current chemical space explored in medicinal chemistry.

For compounds identified as potential BSEP inhibitors in vesicles (i.e., BSEP $\mathrm{IC}_{50}<10$-fold total $\mathrm{C}_{\text {max,ss }}$ ), the guided workflow in the white paper recommends undertaking investigatory studies, which may include assessment of the BEI of TCA in a hepatocyte-based system such as sandwich-cultured hepatocytes. Our data indicate that MPCCs can also be used as a functional hepatocyte-based system for evaluating potential to inhibit canalicular efflux of bile salts, supporting the need for hepatocyte-based models to overcome limitations of the vesicular inhibition assay alone. In addition, MPCCs have been shown to improve in vitro-in vivo correlation by detecting primary and secondary metabolites (DaSilva et al., 2018).

Beyond the use of a hepatocyte-based model to evaluate inhibition of TCA efflux, the guided workflow suggests consideration of inhibition of other bile acid transporters, including MRPs. MRP-mediated efflux has been proposed as a compensatory pathway used by hepatocytes to efflux accumulated intracellular bile salts into the plasma when BSEP is inhibited (Köck and Brouwer, 2012). The results in Figure 4 show that inhibition of MRP2-4 does not increase specificity of DILI predictions beyond BSEP inhibition alone and exhibits lower positive predictive value. Previous reports lacked alignment on the value of MRP inhibition in DILI risk assessment (Morgan et al., 2013; Köck et al., 2014; Yucha et al., 2017). One caveat to the MRP analyses is that probe substrates used in the MRP inhibition studies were not bile acids, and we cannot exclude potential differences in inhibition profiles due to substratedependent inhibition. It is not unexpected that inhibition of MRPs, in the absence of BSEP inhibition, does not correlate with DILI risk, as canalicular efflux via BSEP is the rate-determining step in bile salt excretion. Furthermore, although clinical liver injury data are lacking for patients with polymorphic variants of MRP3/4, patients with polymorphic MRP2 resulting in complete loss of function (Dubin Johnson syndrome) do not exhibit increased incidence of liver injury (KullakUblick et al., 2004)(. Although inhibition of BSEP and MRPs could result in increases in intracellular concentrations of bile acids due to inhibition of both canalicular and sinusoidal efflux pathways, Ost $\alpha / \beta$ may provide an additional efflux pathway to lower concentrations inside hepatocytes (Ballatori et al., 2005; Li et al., 2007; Zhang et al., 2017).

Taken together, our data support a two-tiered approach to limit compounds with potential BSEP inhibition liabilities that enter clinical development (Fig. 10). Early screening can be completed in membrane vesicles. In instances in which a compound's $\mathrm{IC}_{50}$ is above the empirical cutoff established, assessment of TCA BEI inhibition potential can be conducted in MPCCs to further explore whether BSEP inhibition is of significance in vivo. Proactive use of this paradigm at Merck has allowed 


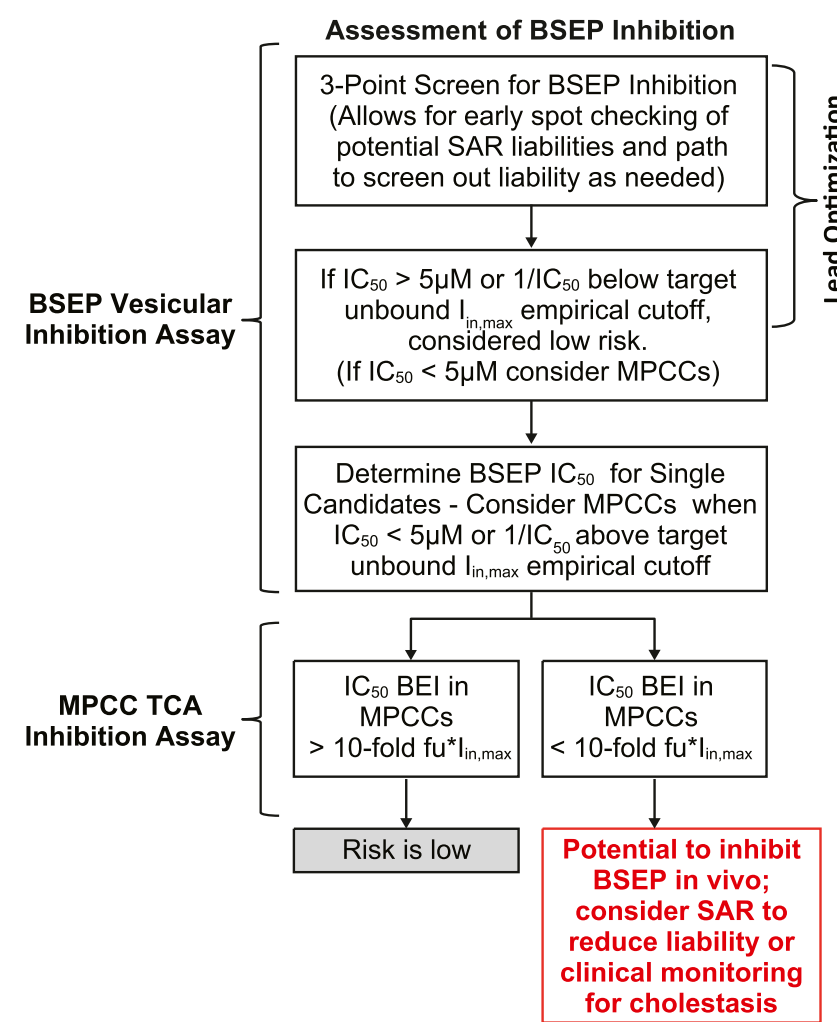

Fig. 10. Application of the vesicular BSEP and MPCC inhibition assays in lead optimization. SAR, structure-activity relationship.

us to further de-risk vesicular BSEP inhibition results with MPCCs for several internal programs, resulting in no known instances of clinical BSEP inhibition, as exemplified by the MK-8666 case study. Although initial inhibition studies with MK-8666 in membrane vesicles warranted further experimentation in MPCCs, the TCA BEI $\mathrm{IC}_{50}$ was more than 10 -fold the predicted $\mathrm{fu}^{*} \mathrm{I}_{\mathrm{in} \text {,max }}$. Because $80 \%$ of MK-8666 was metabolized to an AG in MPCCs and the AG did not inhibit BSEP in the BSEP vesicular assay, metabolism of MK-8666 was a likely cause for the lack of inhibition of TCA transport in the MPCCs. Supporting this finding, plasma samples from healthy volunteers exhibited no elevation in plasma levels of glycochenodeoxycholic acid (GCDCA) on days 1 and 10 after receiving $800 \mathrm{mg}$ every day of MK-8666 compared with those receiving placebo (data on file). A recent publication by Shang et al. (2020) suggests that an alternative mechanism could contribute to DILI risk for MK-8666: metabolism of the carboxylic moiety forms a reactive intermediate, which covalently modifies proteins.

In instances in which the TCA BEI $\mathrm{IC}_{50}$ in MPCCs is $<10$-fold the $\mathrm{fu}^{*} \mathrm{I}_{\mathrm{in} \text {,max }}$, the test compound may have potential to inhibit BSEP in vivo. Given the lack of a specific biomarker and a translational preclinical model, one could develop structure-activity relationships to screen out the liability in vitro or power a phase I study to include markers of cholestasis to assess toxicity potential early in humans. There are several caveats to this tiered approach. First, only compounds that are above the empirical cutoff in vesicles would be tested in MPCCs. Therefore, compounds forming a BSEP-inhibiting metabolite, such as in the case of troglitazone sulfate (Funk et al., 2001), would be missed. Second, parameters used in the $\mathrm{fu}^{*} \mathrm{I}_{\mathrm{in} \text {,max }}$ calculation assume the worst-case scenario (i.e., $\mathrm{Fa}=1, \mathrm{Ka}=0.1$ minute $^{-1}$ ). When available, measured values should be incorporated into the calculation to enhance the accuracy of the assessment. Third, due to compensatory mechanisms, not all BSEP inhibitors will result in cholestasis. By eliminating compounds based on BSEP inhibition alone, one could halt development of a compound that may not present as cholestatic clinically. In the future, development of a relevant in vivo model that replicates druginduced BSEP inhibition to assess human DILI as well as leveraging in vitro/in vivo models to identify, qualify, and optimize the utility of translational biomarkers for BSEP inhibition for clinical application would be needed to further enhance a de-risking strategy.

\section{Acknowledgments}

We thank Amanda Moore and Okey Ukairo, formerly of Ascendance Bio (Medford, MA), for technical support and scientific insight into this work. We thank Sheri Smith, Richard Gundersdorf, and Yuexia Liang for MPCC metabolite identification support.

\section{Authorship Contributions}

Participated in research design: Hafey, Houle, Sistare, Evers.

Conducted experiments: Hafey, Houle, Shang, Chen, Baudy.

Performed data analysis: Hafey, Houle, Tanis, Knemeyer, Shang, Chen, Baudy, Monroe.

Wrote or contributed to the writing of the manuscript: Hafey, Houle, Tanis, Baudy, Monroe, Sistare, Evers.

\section{References}

Balistreri William F, Bezerra Jorge A, Jansen Peter, Karpen Saul J, Shneider Benjamin L, and Suchy Frederick J (2005) Intrahepatic cholestasis: summary of an American Association for the Study of Liver Diseases single-topic conference. Hepatology 42 (1):222-235, doi: 10.1002/ hep.20729 15898074

Ballatori N, Christian WV, Lee JY, Dawson PA, Soroka CJ, Boyer JL, Madejczyk MS, and Li N (2005) OSTalpha-OSTbeta: a major basolateral bile acid and steroid transporter in human intestinal, renal, and biliary epithelia. Hepatology 42:1270-1279.

Brouwer KL, Keppler D, Hoffmaster KA, Bow DA, Cheng Y, Lai Y, Palm JE, Stieger B, and Evers R; International Transporter Consortium (2013) In vitro methods to support transporter evaluation in drug discovery and development. Clin Pharmacol Ther 94:95-112.

Burbank MG, Burban A, Sharanek A, Weaver RJ, Guguen-Guillouzo C, and Guillouzo A (2016) Early alterations of bile canaliculi dynamics and the rho kinase/myosin light chain kinase pathway are characteristics of drug-induced intrahepatic cholestasis. Drug Metab Dispos 44 $1780-1793$.

Da-Silva F, Boulenc X, Vermet H, Compigne P, Gerbal-Chaloin S, Daujat-Chavanieu M, Klieber S, and Poulin P (2018) Improving prediction of metabolic clearance using quantitative extrapolation of results obtained from human hepatic micropatterned cocultures model and by considering the impact of albumin binding. J Pharm Sci 107:1957-1972.

Dawson S, Stahl S, Paul N, Barber J, and Kenna JG (2012) In vitro inhibition of the bile salt export pump correlates with risk of cholestatic drug-induced liver injury in humans. Drug Metab Dispos 40:130-138

Deferm N, De Vocht T, Qi B, Van Brantegem P, Gijbels E, Vinken M, de Witte P, Bouillon T, and Annaert $P$ (2019) Current insights in the complexities underlying drug-induced cholestasis. Crit Rev Toxicol 49:520-548.

Food and Drug Administration Center for Drug Evaluation and Research (2020) In Vitro Drug Interaction Studies - Cytochrome p 450, Enzyme- and Transporter-Mediated Drug Interactions Guidance for Industry. Clinical Pharmacology.

Funk C, Pantze M, Jehle L, Ponelle C, Scheuermann G, Lazendic M, and Gasser R (2001) Troglitazone-induced intrahepatic cholestasis by an interference with the hepatobiliary export of bile acids in male and female rats. Correlation with the gender difference in troglitazone sulfate formation and the inhibition of the canalicular bile salt export pump (Bsep) by troglitazone and troglitazone sulfate. Toxicology 167:83-98.

Guo Y, Chu X, Parrott NJ, Brouwer KLR, Hsu V, Nagar S, Matsson P, Sharma P, Snoeys J, Sugiyama Y, et al.; International Transporter Consortium (2018) Advancing predictions of tissue and intracellular drug concentrations using in vitro, imaging and physiologically based pharmacokinetic modeling approaches. Clin Pharmacol Ther 104:865-889.

Hirano M, Maeda K, Shitara Y, and Sugiyama Y (2006) Drug-drug interaction between pitavastatin and various drugs via OATP1B1. Drug Metab Dispos 34:1229-1236.

Holt Michael and Ju Cynthia (2010) Drug-induced liver injury. Handb Exp Pharmacol (196):3-27, doi: 10.1007/978-3-642-00663-0_1 20020257.

Hyde AM, Liu Z, Kosjek B, Tan L, Klapars A, Ashley ER, Zhong YL, Alvizo O, Agard NJ, Liu G, et al. (2016) Synthesis of the GPR40 partial agonist MK-8666 through a kinetically controlled dynamic enzymatic ketone reduction. Org Lett 18:5888-5891.

Jara Paloma, Hierro Loreto, Martínez-Fernández Pilar, Alvarez-Doforno Rita, Yánez Francisca, Diaz María C, Camarena Carmen, De la Vega Angela, Frauca Esteban, Muñoz-Bartolo Gema, et al. (2009) Recurrence of bile salt export pump deficiency after liver transplantation. $N$ Engl J Med 361 (14):1359-1367, doi: 10.1056/NEJMoa0901075 19797282.

Kang W, Podtelezhnikov A, Tanis K, Pacchione S, Su M, Bleicher K, Wang Z, Laws G, Griffiths T, Kuhls M, et al. (2020) Development and application of a transcriptomic signature of bioactivation in an advanced in vitro liver model to reduce drug-induced liver injury risk early in the pharmaceutical pipeline. Toxicol Sci. DOI: 10.1093/toxsci/kfaa094 [published ahead of print].

Kenna JG, Taskar KS, Battista C, Bourdet DL, Brouwer KLR, Brouwer KR, Dai D, Funk C, Hafey MJ, Lai Y, et al.; International Transporter Consortium (2018) Can bile salt export pump inhibition testing in drug discovery and development reduce liver injury risk? An international transporter consortium perspective. Clin Pharmacol Ther 104:916-932.

Khetani SR and Bhatia SN (2008) Microscale culture of human liver cells for drug development. Nat Biotechnol 26:120-126. 
Köck K and Brouwer KL (2012) A perspective on efflux transport proteins in the liver. Clin Pharmacol Ther 92:599-612.

Köck K, Ferslew BC, Netterberg I, Yang K, Urban TJ, Swaan PW, Stewart PW, and Brouwer KL (2014) Risk factors for development of cholestatic drug-induced liver injury: inhibition of hepatic basolateral bile acid transporters multidrug resistance-associated proteins 3 and 4. Drug Metab Dispos 42:665-674.

Kratochwil NA, Triyatni M, Mueller MB, Klammers F, Leonard B, Turley D, Schmaler J, Ekiciler A, Molitor B, Walter I, et al. (2018) Simultaneous assessment of clearance, metabolism, induction, and drug-drug interaction potential using a long-term in vitro liver model for a nove hepatitis B virus inhibitor. J Pharmacol Exp Ther 365:237-248.

Kullak-Ublick Gerd A, Stieger Bruno, and Meier Peter J (2004) Enterohepatic bile salt transporter in normal physiology and liver disease. Gastroenterology 126 (1):322-342, doi: 10.1053/ j.gastro.2003.06.005 14699511

Li N, Cui Z, Fang F, Lee JY, and Ballatori N (2007) Heterodimerization, trafficking and membrane topology of the two proteins, Ost alpha and Ost beta, that constitute the organic solute and steroid transporter. Biochem J 407:363-372.

Mita S, Suzuki H, Akita H, Hayashi H, Onuki R, Hofmann AF, and Sugiyama Y (2006) Inhibition of bile acid transport across Nat/taurocholate cotransporting polypeptide (SLC10A1) and bile salt export pump (ABCB 11)-coexpressing LLC-PK1 cells by cholestasis-inducing drugs. Drug Metab Dispos 34:1575-1581.

Monroe J, Tanis K, Podtelezhnikov A, Nguyen T, Mahotka S, Lynch D, Evers R, Palamanda J, Miller R, Pippert T, et al. (2020) Application of a rat liver drug bioactivation transcriptional response assay early in drug development that informs chemically reactive metabolite formation and potential for drug induced liver injury. Toxicol Sci DOI: 10.1093/toxsci/kfaa088 [published ahead of print]

Moore A, Chothe PP, Tsao H, and Hariparsad N (2016) Evaluation of the interplay between uptake transport and CYP3A4 induction in micropatterned cocultured hepatocytes. Drug Metab Dispos 44:1910-1919.

Morgan RE, van Staden CJ, Chen Y, Kalyanaraman N, Kalanzi J, Dunn RT II, Afshari CA, and Hamadeh HK (2013) A multifactorial approach to hepatobiliary transporter assessment enables improved therapeutic compound development. Toxicol Sci 136:216-241.

Morgan Ryan E, Trauner Michael, van Staden Carlo J, Lee Paul H, Ramachandran Bharath, Eschenberg Michael, Afshari Cynthia A, Qualls Charles W Jr., Lightfoot-Dunn Ruth, and Hamadeh Hisham K (2010) Interference with bile salt export pump function is a susceptibility factor for human liver injury in drug development. Toxicol Sci 118 (2):485-500, doi: 10.1093/toxsci/kfq269 20829430

Pedersen JM, Matsson P, Bergström CA, Hoogstraate J, Norén A, LeCluyse EL, and Artursson P (2013) Early identification of clinically relevant drug interactions with the human bile salt export pump (BSEP/ABCB11). Toxicol Sci 136:328-343.

Prueksaritanont T, Chu X, Evers R, Klopfer SO, Caro L, Kothare PA, Dempsey C, Rasmussen S, Houle R, Chan G, et al. (2014) Pitavastatin is a more sensitive and selective organic aniontransporting polypeptide 1B clinical probe than rosuvastatin. Br J Clin Pharmacol 78:587-598.

Ramsden Diane, Tweedie Donald J, Chan Tom S, Taub Mitchell E, and Li Yongmei (2014) Bridging in vitro and in vivo metabolism and transport of faldaprevir in human using a novel cocultured human hepatocyte system, HepatoPac. Drug Metab Dispos 42 (3):394-406, doi 10.1124/dmd.113.055897 24366904

Rodrigues AD, Lai Y, Cvijic ME, Elkin LL, Zvyaga T, and Soars MG (2014) Drug-induced perturbations of the bile acid pool, cholestasis, and hepatotoxicity: mechanistic considerations beyond the direct inhibition of the bile salt export pump. Drug Metab Dispos 42:566-574.

Scheffer G L, Kool M, Heijn M, de Haas M, Pijnenborg A C, Wijnholds J, van Helvoort A, de Jong $\mathrm{M} \mathrm{C}$, Hooijberg J H, Mol C A, et al. (2000) Specific detection of multidrug resistance proteins
MRP1, MRP2, MRP3, MRP5, and MDR3 P-glycoprotein with a panel of monoclonal antibodies. Cancer Res 60 (18):5269-5277 11016657.

Shah Falgun, Leung Louis, Barton Hugh A, Will Yvonne, Rodrigues A David, Greene Nigel, and Aleo Michael D (2015) Setting Clinical Exposure Levels of Concern for Drug-Induced Liver Injury (DILI) Using Mechanistic in vitro Assays. Toxicol Sci 147 (2):500-514, doi: 10.1093/ toxsci/kfv152 26206150.

Shang J, Tschirret-Guth R, Cancilla M, Samuel K, Chen Q, Chobanian HR, Thomas A, Tong W, Josien H, Buevich AV, et al. (2020) Bioactivation of GPR40 agonist MK-8666: formation of protein adducts in vitro from reactive acyl glucuronide and acyl CoA thioester. Chem Res Toxicol 33:191-201.

Sistare FD, Mattes WB, and LeCluyse EL (2016) The promise of new technologies to reduce, refine, or replace animal use while reducing risks of drug induced liver injury in pharmaceutical development. ILAR J 57:186-211.

Swift B, Pfeifer ND, and Brouwer KL (2010) Sandwich-cultured hepatocytes: an in vitro model to evaluate hepatobiliary transporter-based drug interactions and hepatotoxicity. Drug Metab Rev 42:446-471.

Ukairo O, Kanchagar C, Moore A, Shi J, Gaffney J, Aoyama S, Rose K, Krzyzewski S, McGeehan J, Andersen ME, et al. (2013) Long-term stability of primary rat hepatocytes in micropatterned cocultures. J Biochem Mol Toxicol 27:204-212.

Wolf KK, Vora S, Webster LO, Generaux GT, Polli JW, and Brouwer KL (2010) Use of cassette dosing in sandwich-cultured rat and human hepatocytes to identify drugs that inhibit bile acid transport. Toxicol In Vitro 24:297-309.

Xu Q, Liu L, Vu H, Kuhls M, Aslamkhan AG, Liaw A, Yu Y, Kaczor A, Ruth M, Wei C, et al. (2019) Can galactose be converted to glucose in HepG2 cells? Improving the in vitro mitochondrial toxicity assay for the assessment of drug induced liver injury. Chem Res Toxicol 32:1528-1544.

Yang K, Guo C, Woodhead JL, St Claire RL III, Watkins PB, Siler SQ, Howell BA, and Brouwer KLR (2016) Sandwich-cultured hepatocytes as a tool to study drug disposition and drug-induced liver injury. J Pharm Sci 105:443-459.

Yang Kyunghee, Köck Kathleen, Sedykh Alexander, Tropsha Alexander, and Brouwer Kim L R (2013) An updated review on drug-induced cholestasis: mechanisms and investigation of physicochemical properties and pharmacokinetic parameters. J Pharm Sci 102 (9):3037-3057, doi: 10.1002/jps.23584 23653385.

Yoshida K, Maeda K, and Sugiyama Y (2012) Transporter-mediated drug--drug interactions involving OATP substrates: predictions based on in vitro inhibition studies. Clin Pharmacol Ther 91:1053-1064.

Yucha RW, He K, Shi Q, Cai L, Nakashita Y, Xia CQ, and Liao M (2017) In vitro drug-induced liver injury prediction: criteria optimization of efflux transporter $\mathrm{IC}_{50}$ and physicochemical properties. Toxicol Sci 157:487-499.

Zamek-Gliszczynski MJ, Xiong H, Patel NJ, Turncliff RZ, Pollack GM, and Brouwer KL (2003) Pharmacokinetics of 5 (and 6)-carboxy-2', $7^{\prime}$-dichlorofluorescein and its diacetate promoiety in the liver. J Pharmacol Exp Ther 304:801-809.

Zhang Y, Jackson JP, St Claire RL III, Freeman K, Brouwer KR, and Edwards JE (2017) Obeticholic acid, a selective farnesoid $\mathrm{X}$ receptor agonist, regulates bile acid homeostasis in sandwich-cultured human hepatocytes. Pharmacol Res Perspect 5:e00329.

Address correspondence to: Michael J. Hafey, Department of Pharmacokinetics, Pharmacodynamics \& Drug Metabolism (PPDM), Merck \& Co., Inc., PO Box 2000, RY800-B210, Rahway, NJ 07765. E-mail: michael_hafey@merck.com 